\title{
A HOT URANUS ORBITING THE SUPER METAL-RICH STAR HD 77338 AND THE METALLICITY-MASS CONNECTION*
}

\author{
J. S. Jenkins ${ }^{1,2}$, H. R. A. Jones ${ }^{2}$, M. Tuomi ${ }^{2,3}$, F. Murgas ${ }^{4,5}$, S. Hoyer ${ }^{1}$, M. I. Jones ${ }^{1,6}$, J. R. Barnes ${ }^{2}$, Y. V. Pavlenko ${ }^{2,7}$, \\ O. Ivanyuk ${ }^{7}$, P. Rojo ${ }^{1}$, A. Jordán ${ }^{8}$, A. C. Day-Jones ${ }^{1,2}$, M. T. Ruiz ${ }^{1}$, And D. J. Pinfield ${ }^{2}$ \\ ${ }^{1}$ Departamento de Astronomia, Universidad de Chile, Camino el Observatorio 1515, Las Condes, Santiago, Casilla 36-D, Chile; jjenkins@ das.uchile.cl \\ ${ }^{2}$ Center for Astrophysics, University of Hertfordshire, College Lane Campus, Hatfield, Hertfordshire, AL10 9AB, UK \\ ${ }^{3}$ Tuorla Observatory, Department of Physics and Astronomy, University of Turku, Väisäläntie 20, FI-21500, Piikkiö, Finland \\ ${ }^{4}$ Instituto de Astrofísica de Canarias, Via Lactea, E-38205 La Laguna, Tenerife, Spain \\ ${ }^{5}$ Departamento de Astrofísica, Universidad de La Laguna (ULL), E-38206 La Laguna, Tenerife, Spain \\ ${ }^{6}$ European Southern Observatory, Casilla 19001, Santiago, Chile \\ ${ }^{7}$ Main Astronomical Observatory of National Academy of Sciences of Ukraine, 27 Zabolotnoho, Kyiv 127, 03680, Ukraine \\ ${ }^{8}$ Departamento de Astronomía y Astrofísica, Pontificia Universidad Católica de Chile, 7820436 Macul, Santiago, Chile \\ Received 2012 March 20; accepted 2012 December 11; published 2013 March 8
}

\begin{abstract}
We announce the discovery of a low-mass planet orbiting the super metal-rich K0V star HD 77338 as part of our ongoing Calan-Hertfordshire Extrasolar Planet Search. The best-fit planet solution has an orbital period of $5.7361 \pm 0.0015$ days and with a radial velocity semi-amplitude of only $5.96 \pm 1.74 \mathrm{~ms}^{-1}$, we find a minimum mass of $15.9_{-5.3}^{+4.7} M_{\oplus}$. The best-fit eccentricity from this solution is $0.09_{-0.09}^{+0.25}$, and we find agreement for this data set using a Bayesian analysis and a periodogram analysis. We measure a metallicity for the star of $+0.35 \pm 0.06$ dex, whereas another recent work finds $+0.47 \pm 0.05$ dex. Thus HD $77338 b$ is one of the most metal-rich planet-host stars known and the most metal-rich star hosting a sub-Neptune-mass planet. We searched for a transit signature of HD $77338 b$ but none was detected. We also highlight an emerging trend where metallicity and mass seem to correlate at very low masses, a discovery that would be in agreement with the core accretion model of planet formation. The trend appears to show that for Neptune-mass planets and below, higher masses are preferred when the host star is more metal-rich. Also a lower boundary is apparent in the super metal-rich regime where there are no very low mass planets yet discovered in comparison to the sub-solar metallicity regime. A Monte Carlo analysis shows that this low-mass planet desert is statistically significant with the current sample of 36 planets at the $\sim 4.5 \sigma$ level. In addition, results from Kepler strengthen the claim for this paucity of the lowest-mass planets in super metal-rich systems. Finally, this discovery adds to the growing population of low-mass planets around low-mass and metal-rich stars and shows that very low mass planets can now be discovered with a relatively small number of data points using stable instrumentation.
\end{abstract}

Key words: planetary systems - stars: fundamental parameters - stars: individual (HD 77338) - stars: rotation

Online-only material: color figures

\section{INTRODUCTION}

One of the first correlations announced between two parameters that dealt with exoplanets and their host stars was the abundance of heavy elements in the stellar atmospheres. Gonzalez (1997) first noted that all three exoplanet host stars known at that time were overabundant in iron. The paper indicated that the metallicity of these stars was all in the super solar regime. This feature has since been studied by a number of authors, most notably by Fischer \& Valenti (2005) who defined a relationship between the host star metallicity $([\mathrm{Fe} / \mathrm{H}])$ and the probability of a star hosting a gas giant planet.

This metallicity bias was one of the major features that helped to confirm that the core accretion scenario of planet formation (e.g., Ida \& Lin 2004a; Mordasini et al. 2009), coupled with planetary migration (Lin \& Papaloizou 1986), appears to be the dominant mechanism to build these planetary systems. A further validation of the core accretion mechanism again comes from stellar metallicity since it appears that low-mass Neptunes

\footnotetext{
* Based on observations collected at the La Silla Paranal Observatory, ESO (Chile) with the HARPS spectrograph on the ESO $3.6 \mathrm{~m}$ telescope, under the program IDs 079.C-0927, 081.C-0148, 087.C-0368, and 088.C-0662.
}

and super-Earths are not predominantly found around metal-rich stars (Udry et al. 2006).

The Calan-Hertfordshire Extrasolar Planet Search (CHEPS) is a program to mainly monitor metal-rich stars primarily to hunt for short period planets across a wide range of masses and better constrain the statistics of planets around these stars, while also searching for planetary transits of bright and nearby stars in the southern hemisphere. In Jenkins et al. (2008) we discussed the sample selection for the CHEPS, using previous observations made with the ESO-FEROS instrument to measure the chromospheric activity and metallicity of a sample of a few hundred stars. This sample has recently been increased using data discussed in Jenkins et al. (2011) and current metallicity analysis of these stars is still ongoing using our new methods for measuring accurate atomic abundances in stars like the Sun (Pavlenko et al. 2012; Jenkins et al. 2012).

In Jenkins et al. (2009) we published the discovery of an eccentric brown dwarf, or extreme Jovian planet, orbiting the metal-rich star HD 191760, along with new orbits for three other recently discovered southern hemisphere metalrich planets. The CHEPS data have mostly been obtained using the HARPS instrument but significant observing time on Coralie has also recently been acquired to pre-select interesting 
Table 1

HARPS Radial Velocities for HD 77338

\begin{tabular}{|c|c|c|c|}
\hline BJD & RV & $\sigma_{\text {int }}$ & $\sigma_{\text {tot }}$ \\
\hline 2453358.7928045 & -18.11 & 1.66 & 2.67 \\
\hline 2453359.7935070 & -7.53 & 1.89 & 2.83 \\
\hline 2453360.8256229 & -4.32 & 1.61 & 2.65 \\
\hline 2454058.8168860 & -15.45 & 0.49 & 2.16 \\
\hline 2454098.6739885 & -19.44 & 0.71 & 2.22 \\
\hline 2454103.7481653 & -16.52 & 0.73 & 2.15 \\
\hline 2454124.6791307 & -9.52 & 0.46 & 2.15 \\
\hline 2454125.7692682 & -15.18 & 0.55 & 2.17 \\
\hline 2454133.7288993 & -12.63 & 0.55 & 2.17 \\
\hline 2454161.6324334 & -15.91 & 1.09 & 2.37 \\
\hline 2454161.6593658 & -18.24 & 0.46 & 2.15 \\
\hline 2454162.6659105 & -12.47 & 0.49 & 2.16 \\
\hline 2454365.8855969 & -10.19 & 0.74 & 2.23 \\
\hline 2454579.6605011 & -22.23 & 0.78 & 2.24 \\
\hline 2454580.6290112 & -17.33 & 0.72 & 2.22 \\
\hline 2454581.5835519 & -7.64 & 0.65 & 2.20 \\
\hline 2454725.8665876 & -10.20 & 0.82 & 2.25 \\
\hline 2454726.8663334 & -10.79 & 0.72 & 2.22 \\
\hline 2454726.9022721 & -8.34 & 0.58 & 2.18 \\
\hline 2454727.8587883 & -14.39 & 2.09 & 2.96 \\
\hline 2455650.5916958 & -10.35 & 0.65 & 2.20 \\
\hline 2455651.6037866 & -13.69 & 0.75 & 2.23 \\
\hline 2455883.7133405 & -11.57 & 0.76 & 2.24 \\
\hline 2455883.8696951 & -12.36 & 0.59 & 2.18 \\
\hline 2455885.7293282 & -12.72 & 1.03 & 2.34 \\
\hline 2455885.8583195 & -10.69 & 1.02 & 2.33 \\
\hline 2455992.5489188 & -9.95 & 0.48 & 2.16 \\
\hline 2455992.7888091 & -7.77 & 0.65 & 2.20 \\
\hline 2455993.5357474 & -5.27 & 0.48 & 2.15 \\
\hline 2455993.7774660 & -6.55 & 0.56 & 2.17 \\
\hline 2455994.5078761 & -7.82 & 0.60 & 2.18 \\
\hline 2455994.7518050 & -10.03 & 0.68 & 2.21 \\
\hline
\end{tabular}

Note. The $\sigma_{\text {int }}$ and $\sigma_{\text {tot }}$ are the uncertainties on the points before and after adding the estimated activity jitter value shown in Table 3 in quadrature.

new targets for HARPS follow up (see Jenkins \& Jordan 2011).

\section{OBSERVATIONS AND REDUCTION}

All the radial velocity data we present in this work were obtained using the HARPS instrument (Mayor et al. 2003). From empirical data, HARPS has been shown to be radial velocity stable down to the sub $1 \mathrm{~ms}^{-1}$ level (Pepe et al. 2011). HARPS itself is a fiber-fed cross-dispersed echelle spectrograph that employs two $1^{\prime \prime}$ fibers, which in simultaneous Thorium-Argon (ThAr) mode places one fiber on the source (HD 77338 in this case) and one feeds the calibration ThAr lamp to monitor the instrumental drift. The calibration lamp is useful for sub-1 ms ${ }^{-1}$ nightly stability as the instrument itself is stable at the $1 \mathrm{~ms}^{-1}$ level throughout the course of a single night.

We discuss 32 velocity measurements from HARPS in this current work for the star HD 77338 that are shown in Table 1. We follow the procedure of observing our targets over multiple nights in any single run (see Jenkins et al. 2009), which are usually 3-5 days in duration, and with a total integration time of 15 minutes to average over the strongest $p$-mode oscillations in these types of stars (O'Toole et al. 2009).

Our latest data were reduced and velocities extracted in near real time at the telescope using the most up to date version of the HARPS-DRS (Pepe et al. 2004). However, our older data, which were acquired before the latest version of the
Table 2

Elemental Abundances for HD 77338

\begin{tabular}{lcccrr}
\hline \hline Ion & $\log N(\mathrm{X})$ & $\log N(\mathrm{X})_{\odot}$ & {$[\mathrm{X} / \mathrm{H}]$} & {$[\mathrm{X} / \mathrm{Fe}]$} & $N_{l}$ \\
\hline Si I & $-4.00 \pm 0.06$ & $-4.45 \pm 0.05^{\mathrm{a}}$ & 0.45 & 0.10 & 13 \\
Si II & $-3.78 \pm 0.03$ & $-4.45 \pm 0.05^{\mathrm{a}}$ & 0.67 & 0.32 & 2 \\
Ca I & $-5.25 \pm 0.02$ & $-5.64 \pm 0.02^{\mathrm{a}}$ & 0.39 & 0.04 & 12 \\
Ti I & $-6.67 \pm 0.03$ & $-6.98 \pm 0.06^{\mathrm{a}}$ & 0.31 & -0.04 & 29 \\
Ti II & $-6.76 \pm 0.05$ & $-6.98 \pm 0.06^{\mathrm{a}}$ & 0.22 & -0.13 & 27 \\
Cr I & $-6.04 \pm 0.03$ & $-6.33 \pm 0.03^{\mathrm{a}}$ & 0.29 & -0.06 & 38 \\
Fe I & $-4.08 \pm 0.02$ & $-4.42 \pm 0.03^{\mathrm{b}}$ & 0.34 & -0.01 & 130 \\
Fe II & $-4.07 \pm 0.06$ & $-4.42 \pm 0.03^{\mathrm{b}}$ & 0.35 & 0.00 & 18 \\
$\alpha$ & $-5.74 \pm 0.09$ & $-6.08 \pm 0.11$ & 0.34 & -0.01 & 119 \\
\hline
\end{tabular}

Notes.

a Grevesse \& Sauval (1998).

b Pavlenko et al. (2012): $\alpha=$ mean of Si I, Ca I, Ti I, Ti II, and Cr I.

software was installed on site, were re-reduced offline in the ESO Vitacura offices using the latest version of the DRS. This software performs all functions necessary to fully reduce and analyze a radial velocity timeseries, returning barycentric corrected velocities, uncertainties, and a suite of diagnostics to test the line stability of the data set. All activities we analyze were extracted using a custom code developed following our previous experience with this type of chromospheric activity analysis (e.g., Jenkins et al. 2006, 2008, 2011).

\section{HD 77338}

The star HD 77338 is classified as K0IV in the Hipparcos main catalog (Perryman et al. 1997) and has a parallax of $24.54 \pm 1.06$ mas (van Leeuwen 2007), giving rise to a distance of $40.75 \pm 1.76 \mathrm{pc}$. It has an apparent $V$-band magnitude of 8.63 , meaning its distance modulus is 3.05 , and the Johnson $B-V$ color index is 0.833 .

The photometric and astrometric analysis means we can place HD 77338 on an H-R diagram and compare its position to that of model isochrones and isomass tracks. However, in order to do this properly, we must first have a handle on the metallicity. Given that the star is fairly nearby and bright, there have been a number of attempts to pin down its metallicity.

Using FEROS spectroscopy we had previously measured the $[\mathrm{Fe} / \mathrm{H}]$ of HD 77338 to be $+0.25 \pm 0.07$ dex (Jenkins et al. 2008) clearly clarifying that this star is indeed super metal-rich.

Since we now have time series data from HARPS we have also measured the metallicity, and other atomic abundances using this spectra. We normalized each of the one-dimensional spectra and combined all the residual fluxes to gain a high signal-tonoise ratio $(\mathrm{S} / \mathrm{N})$ and high-resolution single HD 77338 optical spectrum. We then applied our new method for measuring selfconsistent abundances from many optical absorption lines for Fe I and Fe II independently (Pavlenko et al. 2012) and find a $\log N(\mathrm{Fe}$ I $)$ of $-4.08 \pm 0.02$ dex and a $\log N(\mathrm{Fe}$ II $)$ of $-4.07 \pm$ 0.06 dex. Although in agreement, the Fe II abundance has a larger uncertainty due to the lower number of lines used to determine this value. The final abundances for iron and other elements, along with the solar values and the number of lines used in the abundance determination for each, are shown in Table 2 .

Taking these two abundances and subtracting off the solar abundance value of -4.42 (Pavlenko et al. 2012) we find an $[\mathrm{Fe} / \mathrm{H}]$ of $+0.35 \pm 0.06 \mathrm{dex}$. This value is in agreement with our previous value to within the uncertainties but is even more metal-rich. It is also in good agreement with other authors, for 


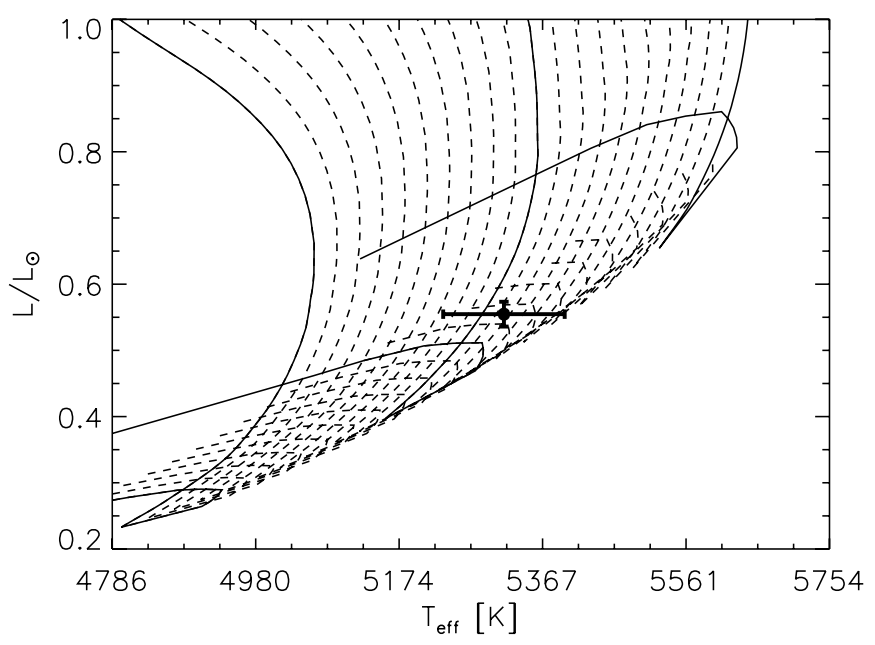

Figure 1. Y2 evolutionary tracks for masses of $1.0,0.9$, and $0.8 M_{\odot}$ are shown by the solid curves from top to bottom. The dashed curves are the higher resolution analysis tracks with a step of $\pm 0.01 M_{\odot}$. The crosshair marks the position of HD 77338.

example, Barbuy \& Grenon (1990) find an $[\mathrm{Fe} / \mathrm{H}]$ of $+0.22 \mathrm{dex}$, Taylor (2005) find a value of $+0.283 \pm 0.051 \mathrm{dex}$, Cenarro et al. (2007) quote a value of $+0.25 \pm 0.10$ dex, and Trevisan et al. (2011) find $+0.47 \pm 0.05$ dex. Finally, we can be sure from these analyses that HD 77338 is a super metal-rich star.

In Figure 1 we show the position of HD 77338 on an H-R diagram (cross hairs), with $T_{\text {eff }}$ on the $x$-axis and $L / L_{\odot}$ on the $y$-axis. Yonsie-Yale (Y2) evolutionary tracks are shown (Demarque et al. 2004), and the analysis method we perform is explained in Jenkins et al. (2009). The important point here is that the solid curves represent three masses of $0.8,0.9$, and $1.0 M_{\odot}$, from bottom to top, and the dashed curves show the tracks at a higher resolution of $0.01 M_{\odot}$ within those three mass regimes. This analysis yields a mass of $0.93 \pm 0.05 M_{\odot}$, a radius of $0.88 \pm 0.04 R_{\odot}$, a $\log g$ of $4.52 \pm 0.06 \mathrm{dex}$, and an age of $3.97_{-3.00}^{+4.23} \mathrm{Gyr}$ for HD 77338 . We note that HD 77338 is not a subgiant star as labeled in Hipparcos, but is a typical metal-rich K-dwarf star. All calculated values for HD 77338 are shown in Table 3.

\section{DOPPLER ANALYSIS}

The radial velocity timeseries of data for HD 77338 show evidence for a signal with a very short period of only 5.74 days. The signal is apparent using two separate analysis methods that we describe here.

\subsection{Periodogram Analysis}

A periodogram search for strong and stable frequencies in the radial velocity data set for HD 77338 reveal a single prominent peak. In Figure 2 (upper plot) we show the periodogram for the HD 77338 data and the strongest peak that relates to the 5.74 day Doppler signal is clear. At present there are still some small peaks around this period, but not with a power close to approaching the 5.74 day period.

Two other peaks are seen in the periodogram, at periods of $\sim 4.5$ days and 1.2 days. We have on occasion observed this star multiple times per night to help break this aliasing, but it will require further observing runs to suppress the power around these frequencies. In fact in our final three night run, we observed the star twice per night and this significantly reduced the power in the 1.2 day signal, indicating this is probably a
Table 3

Stellar Parameters for HD 77338

\begin{tabular}{|c|c|c|}
\hline Parameter & HD 77338 & Reference \\
\hline R.A. J2000 (h:m:s) & $09^{\mathrm{h}} 01^{\mathrm{m}} 12^{\mathrm{s}} .494$ & Perryman et al. (1997) \\
\hline Decl. J2000 (d:m:s) & $-25^{\circ} 31^{\prime} 37^{\prime \prime} .42$ & Perryman et al. (1997) \\
\hline Spectral type & KOIV & Perryman et al. (1997) \\
\hline Spectral type & K0V & This work \\
\hline$B-V$ & 0.833 & Perryman et al. (1997) \\
\hline$V$ & 8.63 & Perryman et al. (1997) \\
\hline Distance (pc) & $40.75 \pm 1.76$ & van Leeuwen (2007) \\
\hline $\log R_{\mathrm{HK}}^{\prime}$ & -5.05 & Jenkins et al. (2011) \\
\hline Hipparcos $N_{\mathrm{obs}}$ & 172 & Perryman et al. (1997) \\
\hline Hipparcos $\sigma$ & 0.013 & Perryman et al. (1997) \\
\hline$\Delta M_{V}$ & 0.224 & Jenkins et al. (2011) \\
\hline$L_{\star} / L_{\odot}$ & $0.55 \pm 0.02$ & This work \\
\hline$M_{\star} / M_{\odot}$ & $0.93 \pm 0.05$ & This work \\
\hline$R_{\star} / R_{\odot}$ & $0.88 \pm 0.04$ & This work \\
\hline$T_{\text {eff }}(\mathrm{K})$ & $5370 \pm 82$ & This work \\
\hline$[\mathrm{Fe} / \mathrm{H}]$ & $0.25 \pm 0.07$ & Jenkins et al. (2008) \\
\hline$[\mathrm{Fe} / \mathrm{H}]$ & $0.35 \pm 0.06$ & This work \\
\hline $\log g$ & $4.52 \pm 0.06$ & This work \\
\hline$U, V, W\left(\mathrm{~km} \mathrm{~s}^{-1}\right)$ & $39.1,-27.7,-24.8$ & Jenkins et al. (2011) \\
\hline$P_{\text {rot }, \mathrm{R}^{\prime} \mathrm{HK}}$ (days) & 45 & This work \\
\hline$P_{\text {rot }, \mathrm{vsini}}$ (days) & 21.7 & This work \\
\hline $\operatorname{Vrot}_{R^{\prime} \mathrm{HK}}\left(\mathrm{km} \mathrm{s}^{-1}\right)$ & 1.1 & This work \\
\hline$v \sin i\left(\mathrm{~km} \mathrm{~s}^{-1}\right)$ & $2.8 \pm 1.5$ & Jenkins et al. (2011) \\
\hline$v \sin i\left(\mathrm{~km} \mathrm{~s}^{-1}\right)$ & $2.33 \pm 0.05$ & This work \\
\hline $\operatorname{Age}_{R^{\prime} \mathrm{HK}}(\mathrm{Gyr})$ & 7.5 & This work \\
\hline Age (Gyr) & $3.97_{-3.00}^{+4.23}$ & This work \\
\hline Jitter- $S_{\mathrm{MW}}\left(\mathrm{m} \mathrm{s}^{-1}\right)$ & $2.10^{-5.00}$ & Isaacson \& Fischer (2010) \\
\hline Jitter-fit $\left(\mathrm{m} \mathrm{s}^{-1}\right)$ & 1.59 & This work \\
\hline
\end{tabular}

sampling frequency. We also note that by looking for power in the sampling, we find some power at periods close to 1 day, further validating the claim that the 1.2 day peak might be a sampling frequency and not a genuine Doppler frequency. The strongest peaks in the sampling frequencies are around 35 days; no significant power is found close to the 5.7 day period of our planet candidate signature.

We ran a false alarm probability (FAP) test by scrambling the velocities with replacement, but retaining the time-stamps from our observations, to test how strong the signal we detect is, in comparison to its emergence being solely through random noise fluctuations. We ran this bootstrap simulation 10,000 times, similar to that in Anglada-Escudé et al. (2012), and checked how often the peak power of the scrambled data was higher than or equal to the power of the observed data. This test revealed that $0.01 \%$ of the time the peak power was larger than the observed data set. This gives us confidence that the signal we detect is robust. The 1\% FAP boundary is shown for reference on the plots. We also note that the FAP registers as $0.02 \%$ if we bin the data points that were observed on the same night.

We make it clear that the signal peak was already significant with only 27 data points, i.e., before our final three night observing run. At that point the FAP registered as $0.02 \%$, and this led us to obtain two radial velocities per night in our last three night run, since we should sample more than half of the planetary candidate orbit in that time. Since the signal power increased significantly from the addition of these six velocities, we believe it is a genuine Doppler reflex motion of the star and not related to a harmonic of our sampling strategy.

The lower plot in Figure 2 shows the periodogram of our residuals after subtraction of our best-fit solution. There is no significant power left in the data after this single planet fit, 

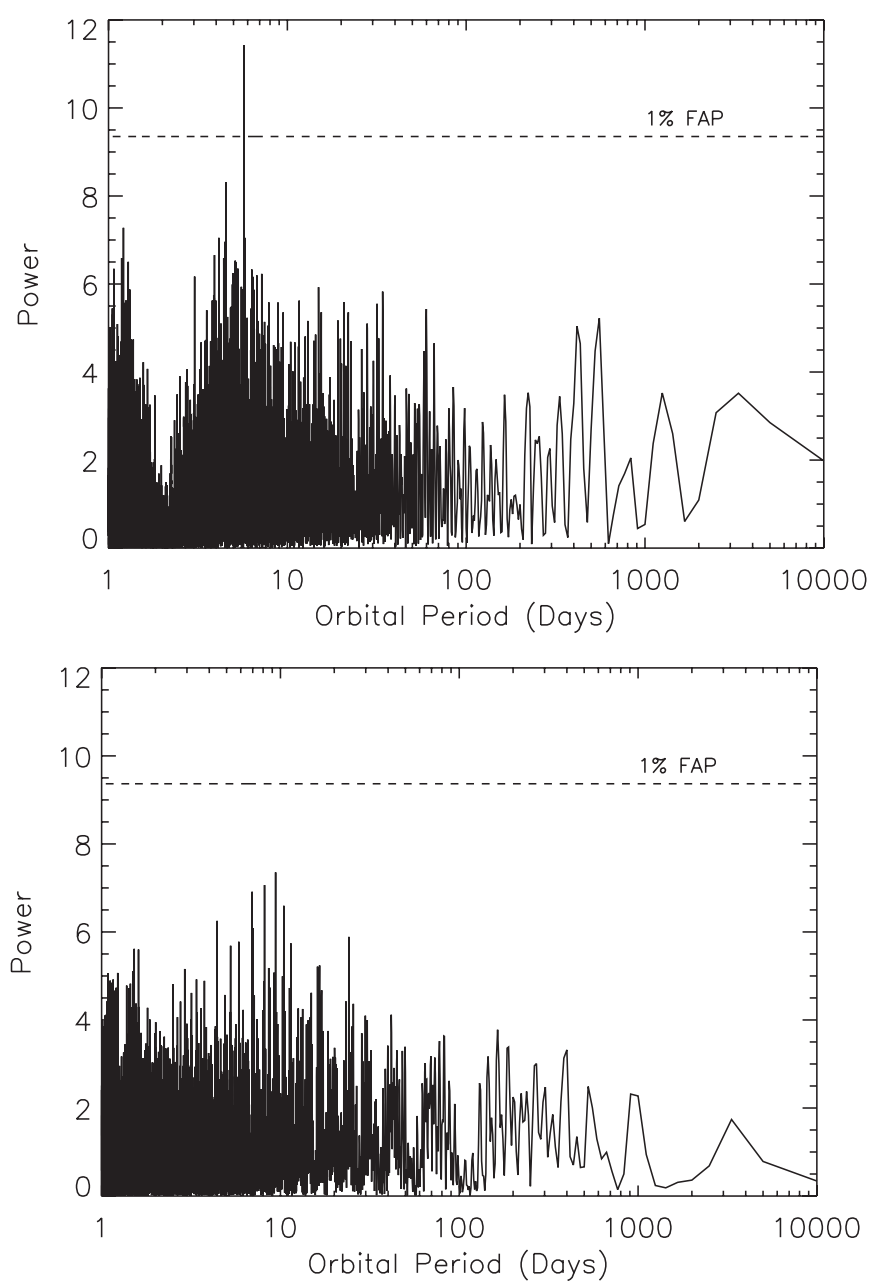

Figure 2. Lomb-Scargle periodogram of the HD 77338 radial velocity data set is shown in the top plot, with the same analysis performed on the residuals to the one-planet fit shown in the lower plot. The dashed line boundaries mark the $1 \%$ FAP limit.

an expected result given the relatively small number of data points we have acquired at present. This also highlights how well the Keplerian fit models the current data set as there is no residual power except white noise. The strongest frequency is found to be around 9.7 days, which could be an emerging planetary signature given the high frequency of multi-planet low-mass systems, however as we show below this is likely due to chromospheric activity.

\subsection{Bayesian Search}

We also run a Bayesian search for signals by assuming that $0,1,2, \ldots$, etc., Keplerian signals best describe the data we have and find the best fit that conforms to our assigned probability threshold. We analyzed the radial velocities using Markov Chain Monte Carlo sampling of the parameter space of each model with a different number of signals. These samplings were performed using the adaptive Metropolis algorithm (Haario et al. 2001; Tuomi et al. 2011; Tuomi 2011). As in Tuomi (2011) and Tuomi et al. (2011) we also used the sample to assess the relative goodness of the different models by calculating their Bayesian probabilities given the data. For a positive detection of $k+1$ signals in the data, we required that the posterior probability of a $k+1$-signal model was 150 times greater than that of a $k$-signal model (e.g., Kass \& Raftery 1995; Feroz et al. 2011) and that the

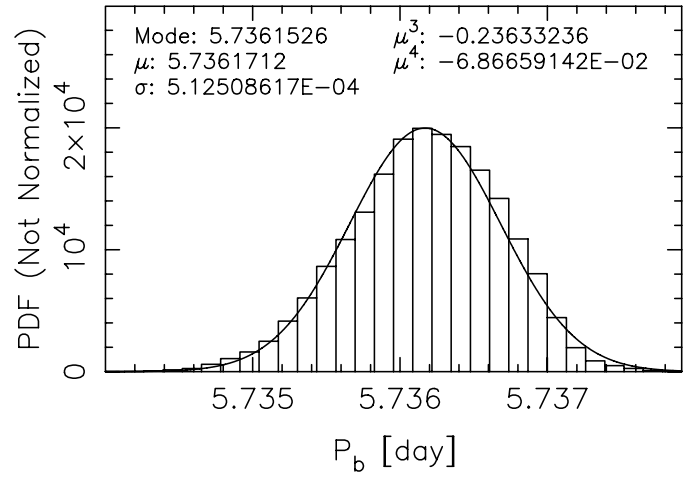

Figure 3. Histogram estimating the probability density function of periods from the Bayesian analysis. Also overplotted by the solid curve is a Gaussian curve with the same mean and sigma as the distribution.

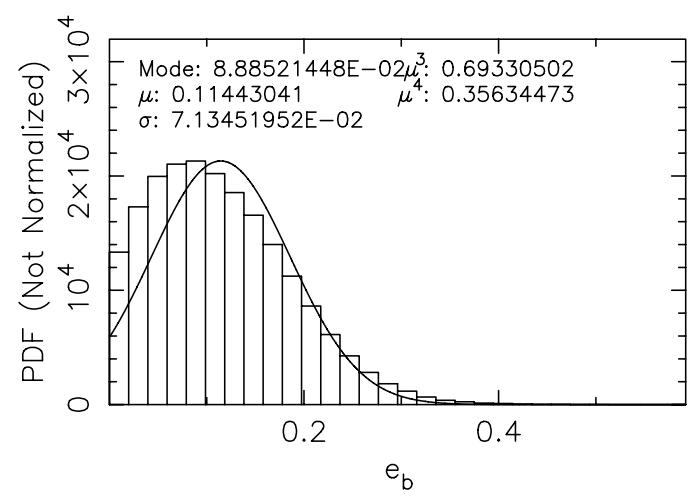

Figure 4. Histogram estimating the probability density function of eccentricities from the Bayesian analysis. Also overplotted by the solid curve is a Gaussian distribution with the same mean and sigma as the histogram.

amplitudes of all the signals were statistically distinguishable from zero.

The Bayesian search we performed using different initial states in the parameter space as starting points of our posterior samplings, quickly located a signal with a period of 5.74 days. This signal satisfied our detection criteria since the probability of no signals is only $6 \times 10^{-8}$, whereas a one-planet model has a probability of essentially unity. The samplings did not reveal any other significant signals in the data. Figure 3 shows the probability density function (PDF) for the returned periods as a histogram. The solid curve is a Gaussian profile with the same characteristics as the histogram, peaking at the measured period, and spread across a confined range of periods. The plot key highlights some of the Gaussian parameters; in particular, the $\sigma$ width of the distribution shows how well constrained the signal is using this technique, as the range of periods here is only at the level of 0.001 days.

Figure 4 shows a similar output PDF but for the possible range of eccentricities. This histogram does not have a Gaussian shape, more Poissonian, peaking around 0.10 or so. Again, a Gaussian profile with parameters from the data is shown for reference. The parameters that describe the Gaussian are shown and the $\sigma$ is now at the level of 0.07 in eccentricity. With a period of less than 6 days, putting the planet closer to the star than the tidal dissipation radius, we expect that the actual curve is probably more circular, but with the relatively small number of data points, we are seeing a more eccentric planetary orbit (O'Toole et al. 2008).

To test the plausibility of the non-zero eccentricity model for this system we ran the Lucy \& Sweeney (1971) test on the data. The test returned a significance, $p$, of $6.7 \%$, showing that the 
Table 4

Maximum a Posteriori Orbital Parameters and their 99\% Credibility Intervals for HD $77338 b$ for Unfixed (Middle Column) and Fixed (Right Column) Eccentricity

\begin{tabular}{lcc}
\hline \hline Parameter & Model 1 & $\begin{array}{c}\text { Model } 2 \\
\text { Fixed Eccentricity }\end{array}$ \\
\hline Orbital period $P$ (days) & Unfixed Eccentricity & $5.7361[5.7345,5.7374]$ \\
Velocity amplitude $K\left(\mathrm{~m} \mathrm{~s}^{-1}\right)$ & $5.7361[5.7346,5.7376]$ & $5.96[4.27,7.62]$ \\
Eccentricity $e$ & $5.96[4.22,7.70]$ & $0.0[\mathrm{fixed}]$ \\
$\omega(\mathrm{rad})$ & $0.09[0,0.34]$ & $0.0[\mathrm{fixed}]$ \\
$M_{0}(\mathrm{rad})$ & $3.9[0,2 \pi]$ & $5.8[0,2 \pi]$ \\
$m \sin i\left(M_{\oplus}\right)$ & $3.5[0,2 \pi]$ & $15.5[11.1,20.9]$ \\
Semimajor axis $a(\mathrm{AU})$ & $15.9[10.6,20.6]$ & $0.0611[0.0581,0.0642]$ \\
$\gamma\left(\mathrm{m} \mathrm{s}^{-1}\right)$ & $0.0614[0.0580,0.0645]$ & $-0.66[-1.79,0.36]$ \\
$\operatorname{rms}\left(\mathrm{m} \mathrm{s}^{-1}\right)$ & $-0.64[-1.81,0.53]$ & 1.71 \\
$N_{\text {Obs }}$ & 1.74 & 32
\end{tabular}

Notes. The uncertainties on the $m \sin i$ and semimajor axis consider the uncertainties on our stellar mass estimate. The $\gamma$ offset is the value after subtracting off the mean of the data set.

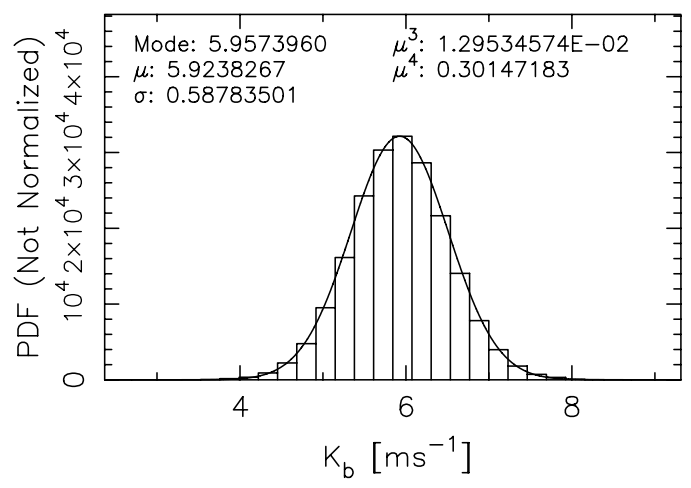

Figure 5. Histogram estimating the probability density function of semiamplitudes for the data from the Bayesian analysis. Also overplotted by the solid curve is a Gaussian distribution with a matching mean and sigma to that of the histogram.

eccentric orbit is not significant at the $5 \%$ threshold level put forward by Lucy and Sweeney. Due to this result we also show a model fit that has the eccentricity fixed to zero in Table 4.

The final PDF we are interested in is shown in Figure 5, which is for the semi-amplitude of the Doppler signal and shows, in accordance to our detection criterion, how it differs significantly from zero. The peak of the histogram is around $6.0 \mathrm{~ms}^{-1}$ and the histogram again has a Gaussian shape, modeled by the solid curve and from the key we see that the $\sigma$ width is less than $60 \mathrm{~cm} \mathrm{~s}^{-1}$. From this semi-amplitude and estimated stellar mass we directly derive the minimum mass of the orbiting planet, finding a value of $15.9 M_{\oplus}$.

The final Keplerian curve describing this Doppler signal is shown in Figure 6, phased to the 5.74 day period of the planet. It is clear that the data describe the periodicity well. The uncertainties on the data points do not include estimated activity-related jitter. Including the estimated jitter shown in Table 3 does not change any of the final orbital values since we treat the excess noise in the data as another free parameter in our statistical model (see Tuomi et al. 2011). Our sampling strategy of observing stars over consecutive nights helps to uncover these short periods, giving us efficient access to low-mass planets on short period orbits such as HD 77338b. We also note that the $\gamma$ values we quote are with respect to the local mean of the data in Table 1.

One issue we have to contest with in this data set is the interdependency of the velocities we observed in a single night.

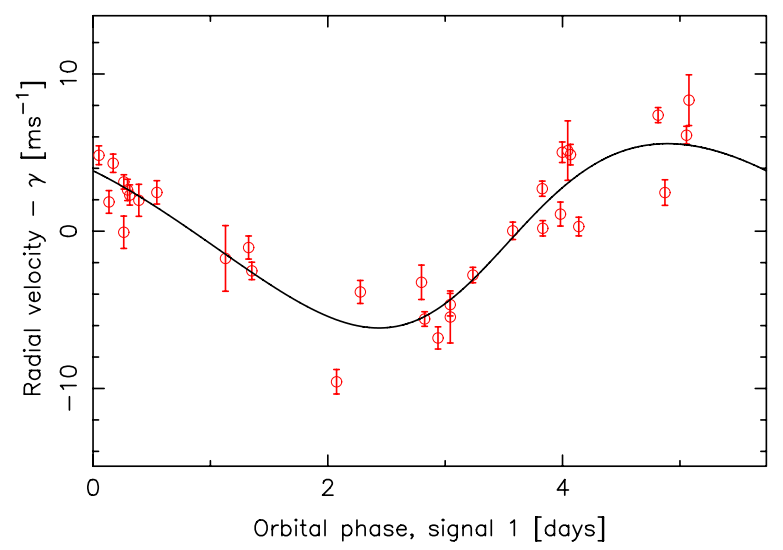

Figure 6. Phase folded best-fit Keplerian orbital solution found using our Bayesian analysis method is shown by the solid curve.

(A color version of this figure is available in the online journal.)

On seven of the nights more than one radial velocity point was measured, meaning in the strictest sense, the data are not independent. However, to take this into consideration we have recently developed a model that employs an Auto-Regression and Moving Average (ARMA) algorithm to model the noise as an input prior into our full Bayesian model (for details and performance of the ARMA model we refer the reader to Tuomi et al. 2013). We ran our ARMA Bayesian model on this data set and find the solution to be statistically similar to the ARMA free algorithm. We find that the moving average components become statistically indistinguishable from zero. Therefore, there are no correlations in the noise, at least not large enough for us to detect.

\section{ACTIVITY INDICATORS}

It has been well established that the CCF bisector span (BIS) can correlate with radial velocity timeseries data, indicating that a given frequency detected in the velocities is not due to a genuine Doppler motion, but originates from the star itself, from processes that induce line asymmetries in the optical spectra of stars (e.g., Queloz et al. 2001; Santos et al. 2010). Asymmetries can arise from spot patterns rotating across the disk of the star, blocking some of the light, and leading to misshapen spectral lines that correlate with the stellar rotation period. When fixed Gaussian profiles are fit to the final cross-correlation functions (CCFs), they do not model the misshapen profile, and so the 

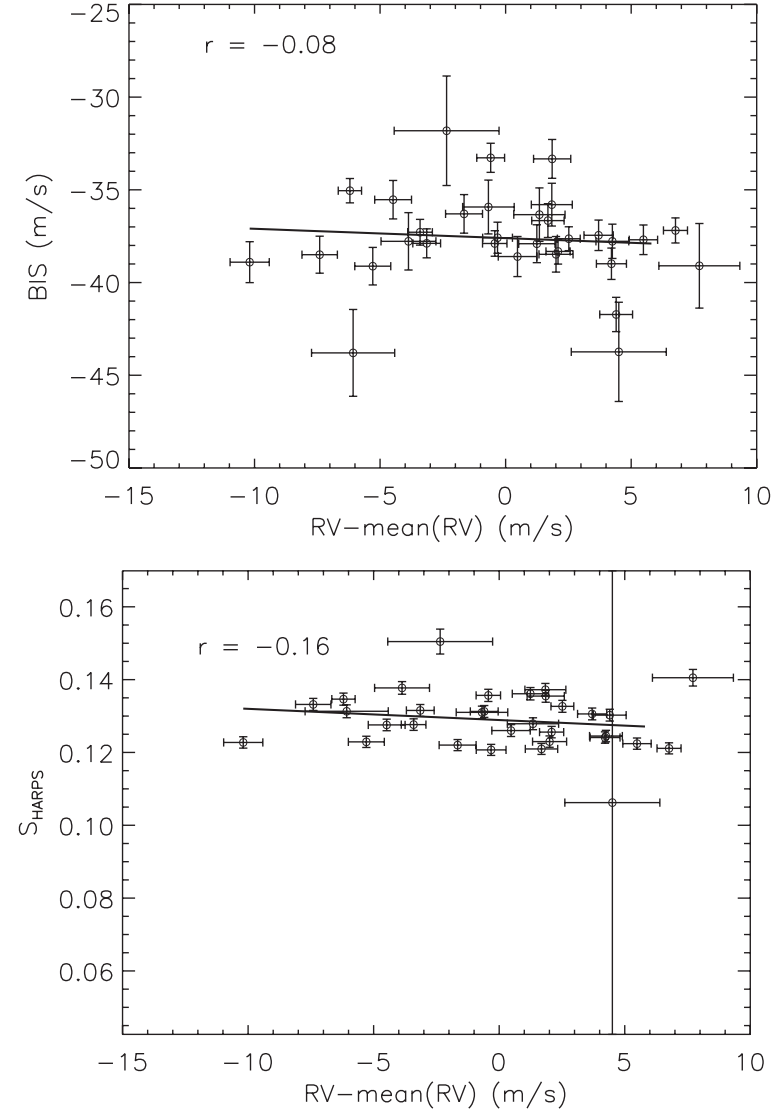

Figure 7. Correlations between the radial velocities and the activity indicators that can be drawn from the HARPS reduced spectra. The top panel shows the CCF bisector span against the radial velocities and the lower panel shows the activity $S$-indices for HD 77338 as a function of the velocities. The solid lines show the best-fit linear correlations between the parameters and the correlation coefficients are highlighted in the panels.

centroid of the Gaussian "wobbles" around the true value, in phase with the stars rotation period.

One test of a true Doppler signal that is not related to stellar activity or velocity fields within the star is to look for correlations between the BIS and the radial velocity timeseries. In the top panel of Figure 7 we show the radial velocities against the BIS for HD 77339. The solid line is the best linear fit to the data and reveals that there is no correlation between the two parameters. To place this on a statistical footing we also show the Pearson linear correlation coefficient $(r)$ within the plot, and the value of -0.08 shows that there is no statistical correlation between the radial velocities and the CCF BIS values, highlighting that the signal we detect is not due to line asymmetries in the star. Also a periodogram analysis does not reveal any power around a period of 5.7 days.

As mentioned above, magnetically active regions (spots) rotating across the star disk due to the rotation of the star can lead to frequencies appearing in the radial velocities. Given that we measured the star to have a $\log R_{\mathrm{HK}}$ of only -5.05 , we would not expect the star to be very spotty and also we would not expect a rotational period as short as $\sim 6$ days. Also, our $v \sin i$ measurement is very small $\left(2.33 \pm 0.05 \mathrm{~km} \mathrm{~s}^{-1}\right)$, confirming that a longer rotational period is preferred for HD 77338. In any case we still test if the chromospheric activity indices correlate with the radial velocities for HD 77338.

The lower panel of Figure 7 shows the HARPS activity $S$ indices against the radial velocities. These indices were

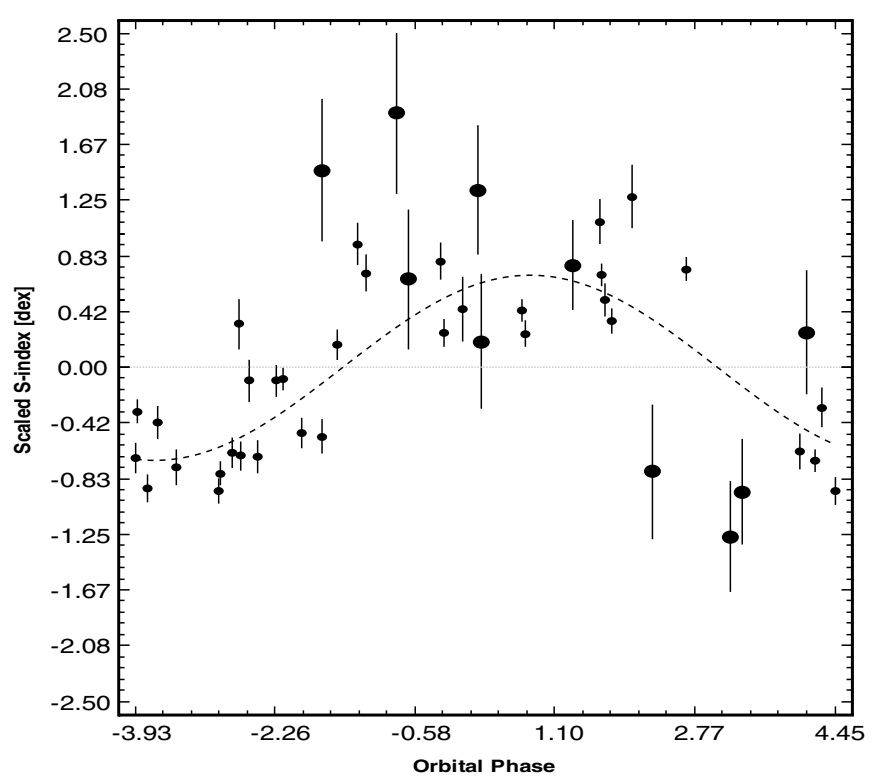

Figure 8. Scaled $S$-indices from both HARPS (small uncertainties) and Coralie (large uncertainties) show possible spot modulation phased to a period of around 9 days.

computed directly from the extracted one-dimensional HARPSDRS spectra, following the methods we have previously laid out in detail in Jenkins et al. (2006, 2008, 2011). The plot shows there is no apparent correlation between the two properties. The solid linear fit has only a small slope and we find the correlation coefficient, $r$, to have a value of -0.16 , showing no significant relationship between the activities and the radial velocities. Again, a periodogram analysis shows no significant power anywhere across all periods. We do note that this fit is unweighted, and the lower activity point with low $\mathrm{S} / \mathrm{N}$ and large uncertainties is probably pulling the slope lower than it should be given the rest of the data set.

It is also unlikely that rotationally modulated spot patterns could give rise to the detected radial velocity variations since the signal has been stable for at least seven years. Also the measured rotational velocity is very low, meaning a rotation period of less than 6 days would require an inclination angle of $\sim 18^{\circ}$, assuming a spherical rotating body. Such a low inclination has a probability of only $4.9 \%$. The very low chromospheric activity would also argue against a rotational period of less than 6 days, along with the presence of large spot patterns. Given that the star is of K spectral type, we expect a lower contrast between the stellar photosphere and spots, in comparison to typical Sun-like G-dwarfs, meaning that the radial-velocity-induced variation from any stable spot patterns would be lower in comparison to more Sun-like stars.

A final test we were able to make was done by including the limited number of Coralie activity indices we have for this star. Inclusion of the Coralie radial velocities does not yield any additional confirmation of the reality of the Doppler signal since the uncertainties from our pipeline are larger than the amplitude of the signal; however, the $S$ indices at this resolution and $\mathrm{S} / \mathrm{N}$ are accurate enough to help test for spot modulation. In Figure 8 we show the combined HARPS and Coralie $S$-index time series phased to the period of the strongest peak in the activity periodogram of 9.001 days (Figure 8). HARPS data alone favor a 10 day peak signal, with this 9 day signal the second strongest peak, but with the inclusion of Coralie data, the 9 day frequency peak is the strongest. The period of this 
possible magnetic modulation is far from the Doppler velocity period yet it is found to be close to where the strongest peak in the residuals of the Keplerian fit to the radial velocities is found, as noted above. This indicates that the peak in the residuals of the best fit to the radial velocities is likely related to spot modulation and not a secondary planetary companion.

In addition to correlations between the BIS and $S$ indices with the radial velocities, we also test for correlations between the CCF full width at half-maximum (FWHM) or the contrast of the CCF with the velocities. The FWHM and the contrast of the CCF has also been shown to be useful indicators of line variations, but at a lower level of significance for these types of stars (Boisse et al. 2011). Our correlation analyses found no strong correlations between these parameters and the velocities, strengthening the case for the reality of a Doppler signal. The final conclusion that can be drawn from analysis of the properties of the CCF and the chromospheric activity values is that the short period signal we have discovered in the radial velocity timeseries of HD 77338 is from a genuine short period planet orbiting the star, with a low minimum mass that gives rise to the possibility of a planet with a non-negligible rocky mass fraction. The final parameters describing the orbit of HD $77338 b$ are shown in Table 4 for our best fit (top) and with a fixed eccentricity of zero (bottom).

\section{TRANSIT FOLLOW-UP}

Short period planets orbiting bright stars can potentially yield transit dips in the light profile of their host stars, and by doing so, can lead to detailed follow-up of atmospheric physics, such as those performed on HD 209458b, HD 189733b, and GJ $1214 b$ (e.g., Pont et al. 2008; Swain et al. 2009; Beaulieu et al. 2010; Bean et al. 2011). Since HD 77338 is bright (but not too bright to preclude transit detection using $1 \mathrm{~m}$ class telescopes, e.g., Jehin et al. 2010), having a $V$-band magnitude of 8.63 , and the planet has an orbital period of less than 6 days, giving rise to a transit probability of $6.4 \%$, we performed photometric monitoring around the predicted time of transit from the orbital solution to test if HD $77338 b$ could be added to this class of bright transiting planets.

We performed the search for the transit of this planet using our current best-fit solution for the system parameters; however, we suspect that a solution with a more circular orbit may be closer to the true nature of the HD 77338 system. Therefore, assuming a circular orbit and an inclination angle of $90^{\circ}$, we can then estimate the transit duration of the planet from

$$
t_{\mathrm{circ}}=\frac{P}{\pi} \arcsin \left(\frac{\sqrt{\left(R_{\star}+R_{p}\right)^{2}}}{a}\right),
$$

where $P$ is the orbital period, $R_{\star}$ and $R_{p}$ are the stellar and planetary radii, respectively, and $a$ again is the semimajor axis. Assuming a planetary radii commensurate with that of Uranus, then the transit duration is predicted to be $2.9 \mathrm{hr}$. Even if the planetary radius is upward of a Jupiter radius, the transit duration is still estimated to be $2.9 \mathrm{hr}$ as it contributes such a small amount to the net radii in comparison to the star.

The star was observed on 2012 March 25 and we used the $1 \mathrm{~m}$ Cerro Tololo Inter-American Observatory (CTIO) Telescope, along with the Y4KCam instrument, located at the CTIO observatory, in Directors Discretionary Time, to gain the necessary photometric precision to search for a transit of the discovered planet. Y4KCam is a $4064 \times 4064$ pixel CCD camera

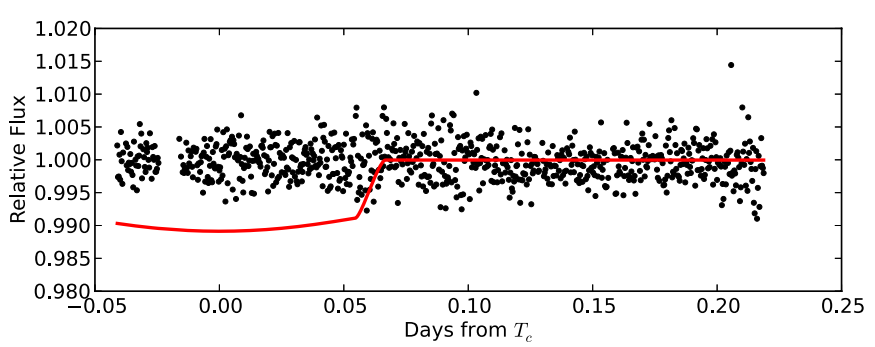

Figure 9. Photometric light curve of HD 77338 covering the predicted time the planet would pass in front of the host star. The red curve is the model prediction for how long and how deep the transit should have been if it was observed.

(A color version of this figure is available in the online journal.)

with a field of view of $20 \times 20 \operatorname{arcmin}^{2}$ and a pixel scale of 0 .'289 pixel $^{-1}$. The standard readout time of the camera is $46 \mathrm{~s}$, which we reduce down to $16 \mathrm{~s}$ by employing a spatial binning of $2 \times 2$.

We employed a strategy of observing the star at integration times of between 7 and $10 \mathrm{~s}$ each, with the telescope operating at heavy defocus to get point spread functions (PSFs) with a typical radius of $\sim 28$ pixels. We obtained 786 data points with typical counts in the range 20,000-25,000, across a time baseline of over $6 \mathrm{hr}$, crossing the predicted center of transit and the predicted egress of planet off the limb of HD 77338. The observed images were then processed using a custom-made pipeline that performs the necessary trimming, bias subtraction, and flat-field correction to prepare the images for final analysis.

Figure 9 shows the normalized photometric light curve of HD 77338 when the planet was estimated to pass in front of the star. We find no suggestion of a transit around the predicted time of center of transit of JD 2456012.527888. The rms scatter of our unbinned photometric data is 0.0028 meaning we could significantly detect any transit securely with a depth of only $0.008 \%$ below the continuum level.

For comparison, the red curve highlights the predicted depth and time of transit for HD $77338 b$ given the orbital solution we found from the radial velocity data and a planetary radius commensurate with that of Saturn. Since no transit dip was detected, we can say that either the inclination of the system is so low that the planet does not pass in front of HD 77338 in our line-of-sight viewing angle, and/or the orbital solution we present is not secure enough to pin down a transit detection. The first of these may be the case and is something we cannot do anything about, however the second can be remedied with more radial velocity data, which we are still acquiring to search for additional planets in the system. The Lucy and Sweeney test we performed also indicated that a circular orbit cannot be ruled out and therefore we should again search for a transit of HD $77338 b$ using the circular orbital solution to test whether or not we can detect any occultation of the planet.

\section{KINEMATIC MOTION}

The Hipparcos proper motion of this dwarf star is $41.13 \pm$ 0.67 mas in $\mu_{\mathrm{ra}}$ and $-270.80 \pm 0.67$ mas in $\mu_{\mathrm{dec}}$, meaning it is classed as a high proper motion star. We used these values to measure the three kinematic velocity components $(U, V, W$; Jenkins et al. 2011), and find velocities of $39.1 \pm 2.0,-27.7 \pm$ 1.8 , and $-24.8 \pm 1.3 \mathrm{~km} \mathrm{~s}^{-1}$, respectively.

Figure 10 shows the distribution of all the stars in Jenkins et al. in the $U V$ plane, along with the position of HD 77338 (filled red circle), the boundaries of the young (rectangle), and old (ellipse) disks from Eggen (1969). HD 77338 is found close 


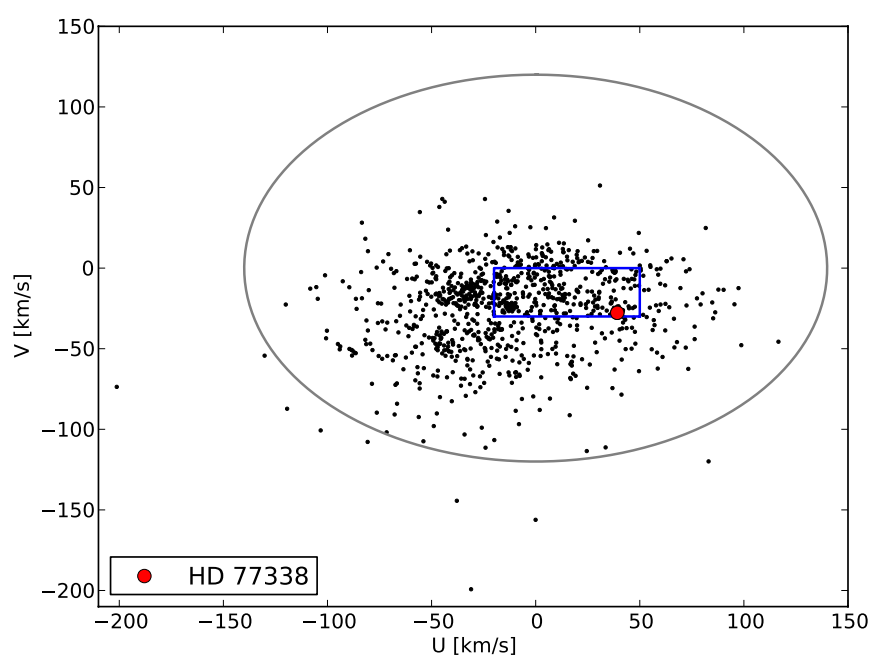

Figure 10. Plot in UV space of the kinematic motion of all stars in Jenkins et al. (2011). The filled red point marks the position of HD 77338. The solid box and solid ellipse mark the regions of the young and old disk, respectively, from Eggen (1969).

(A color version of this figure is available in the online journal.)

to the boundary between the young and old disk. Figure 11 shows a Toomre diagram for the same sample and we see here that HD 77338 is consistent with either the thin or thick disks, in agreement with the $U V$ plane kinematics. We also show the positions of all other hosts stars that have planets with minimum masses $\leqslant 0.05 M_{\mathrm{J}}$ with the colors of the points representing the metallicity of the host star, from metal-poor being blue through to red at the metal-rich end.

In comparison to the other low-mass planet hosts, HD 77338 is fairly high in the energy plane and very central in the $V$ kinematic velocity plane, along with being the most metalrich. As expected, most of the host stars are located around the region that contains mostly thin disk stars, with a few probable intermediate and/or thick disk hosts. However, the two stars HD 4308 and HD 20794 are found to have kinematics that place them well in the thick disk region and encroaching on the halo proxy perimeter. Also the blue colors of these two show they are metal-poor stars which fits in with their kinematics, whereas the hosts with kinematics that place them in the thin disk have a range of metallicities from really metal-poor through to super metal-rich. These two thick disk hosts have very eccentric orbits around the galactic center (Figure 12; code taken from Scholz et al. 1996) which helps to confirm their membership of the thick disk since older stellar populations tend to have eccentric orbits and high velocities. For example, halo stars in general have very eccentric orbits and attain velocities above $180 \mathrm{~km} \mathrm{~s}^{-1}$ (Nissen \& Schuster 2010) in the Toomre diagram. If these stars are indeed bonafide members of the thick disk then this helps to show that low-mass planets were probably forming early in the formation of the galaxy, if the thick disk was formed through some hierarchal merger or heating process (Statler 1988; Abadi et al. 2003; Villalobos \& Helmi 2008; Brook et al. 2004). However, other recent studies refute the existence of a thick disk being born in this fashion (Bovy et al. 2012a, 2012b).

To further test if HD 77338 is also a thick disk member we perform an analysis of its orbital motion through the galaxy. Figure 13 shows the orbital plots for HD 77338 (top four panels) and we see that it has a fairly eccentric orbit and it travels to $\pm 0.2 \mathrm{kpc}$ above and below the plane of the galaxy. The lower

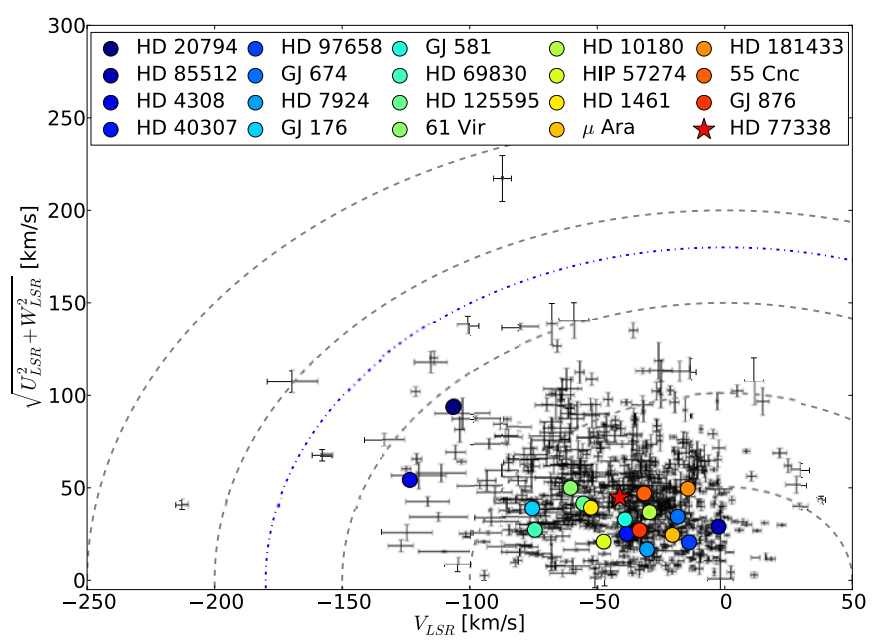

Figure 11. Toomre diagram for the sample of stars in Jenkins et al. (2011) (black points). The colored star corresponds to HD 77338 and the other colored circles are all radial velocity detected planets with sub-Neptune masses and with colors ordered from the most metal-poor (blue) to the most metal-rich (red). The data were taken from the Exoplanet Database as of 2012 February. The dashed lines mark regions of constant kinetic energy.

(A color version of this figure is available in the online journal.)

panels show the same data for the Sun, a typical thin disk star, and we clearly see the circular orbit around the galaxy. Also, it only travels half of the distance in $Z$, above and below the galactic plane, in comparison to HD 77338. These points may indicate that HD 77338 is actually a metal-rich member of the intermediate/thick disk, rather than the thin disk, but thin disk membership cannot be ruled out.

The ratio of $\alpha$ elements in comparison to iron can allow one to distinguish between different stellar populations in some cases. To parameterize the $\alpha$ abundance of HD 77338 we used the $\alpha$ elements shown in Table 2. In general, the measured abundances depend on the adopted microturbulent velocity, $V_{t}$ (see Pavlenko et al. 2012). Using the criteria of the absence of any dependence between $\log N(\mathrm{Fe} \mathrm{I})$ and $\log N(\mathrm{Fe}$ II $)$ against the excitation potential $E^{\prime \prime}$ to select the best absorption lines, we found the best fits to the observed profiles of the Fe I and Fe II ions with a $V_{t}=0.5 \mathrm{~km} \mathrm{~s}^{-1}$. This value was then adopted for the determination of all abundances listed in Table 2:

$$
[\alpha / \mathrm{Fe}]=[\alpha / \mathrm{H}]-[\mathrm{Fe} / \mathrm{H}]
$$

We used the mean of the elements in Table 2 to obtain a $\log N(\alpha)$ value of $-5.74 \pm 0.09 \mathrm{dex}$ for HD 77338, and a solar value of $-6.08 \pm 0.11$ dex, giving rise to an $[\alpha / \mathrm{H}]$ of +0.34 dex. By application of Equation (2) we get the final $[\alpha / \mathrm{Fe}]$ value of -0.01 dex.

We find no real enhancement of the $\alpha$ abundance for a star of such metallicity. Bensby et al. (2005) show abundance trends of such elements and the values of our elements agree well with both the thin and thick disk stars for a super metal-rich star. In fact, Bensby et al. show there is no significant enhancement of most $\alpha$ elements for thick disk metal-rich stars in comparison to metal-rich stars from the thin disk. The conclusion that can be drawn from the kinematics and abundance analyses is that HD 77338 is most likely a thin disk star, or in an intermediate region between the thin and thick disks.

\subsection{Elements}

The $[\mathrm{X} / \mathrm{H}]$ abundances for all the $\alpha$ elements we analyzed are much higher than the solar values, in agreement with the 

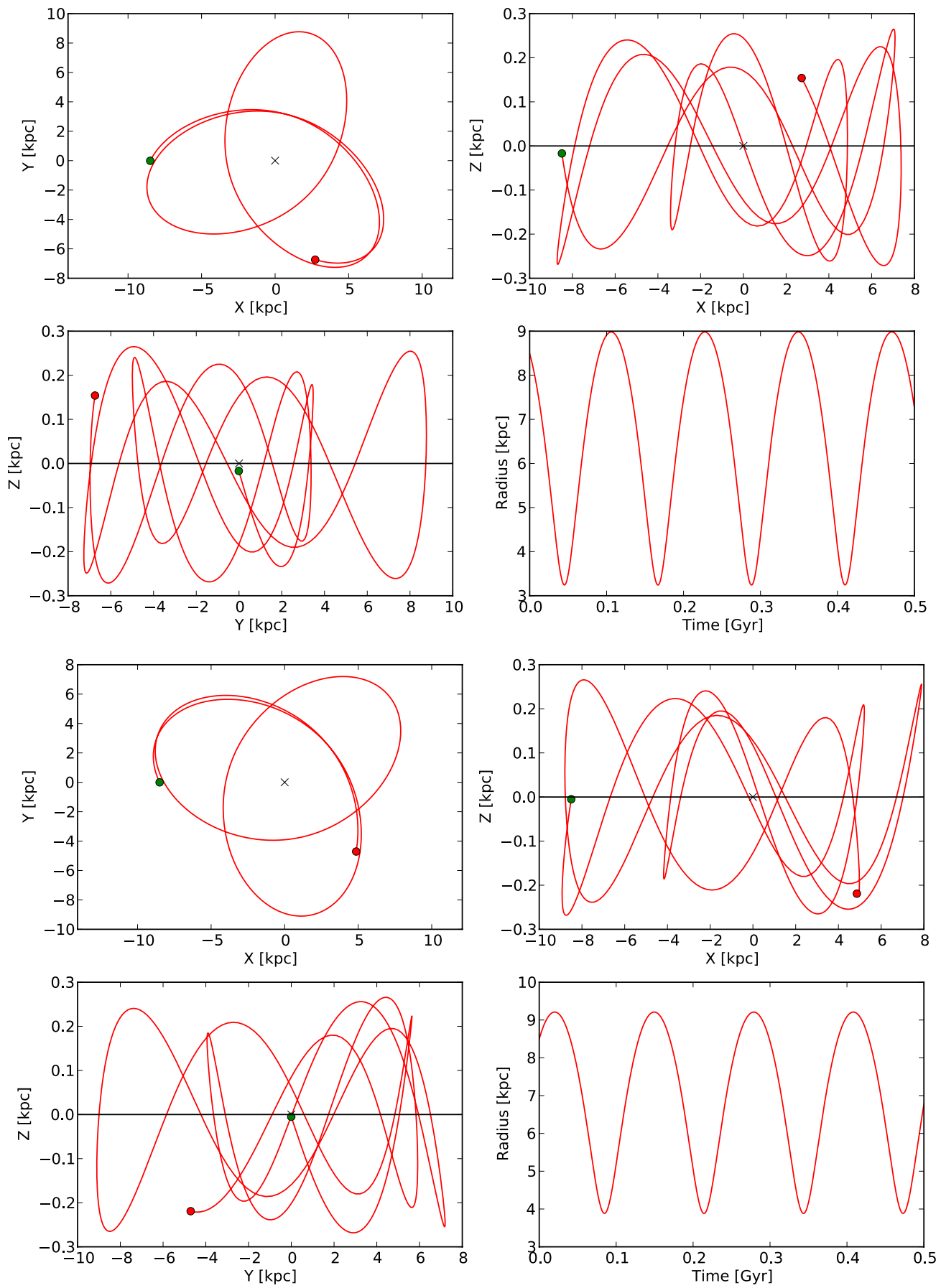

Figure 12. Calculated orbital motion of HD 4308 (top four panels) and HD 20794 (lower four panels) through the galaxy. Both show fairly eccentric orbits and are probably thick disk stars.

(A color version of this figure is available in the online journal.)

super metal-rich nature of HD 77338. The silicon abundance is significantly enhanced if we consider both the neutral and ionized silicon lines; however, as can be seen from Table 2 the Si II analysis only employs 2 useful lines, in comparison to 13 for the Si I abundance. Recently, Trevisan et al. (2011) also found a high silicon abundance for HD 77338 of $+0.47 \pm 0.05$ dex, in good agreement with the value we find here.

Given that silicon is enhanced in general for thin and thick disk stars in comparison to the amount of iron, it becomes a very important ingredient in planet formation models. Large amounts of silicon, and other elements, in the interiors of stars, and by association, the chemical make up of proto-planetary disks, mean a higher disk surface density in general. Changing metallicity, and hence disk surface density, can have a profound impact on planet formation affecting both the masses of planets that can form and also by how much they can migrate (see Mordasini et al. 2012). Metal-rich systems like HD 77338 generally give rise to gas giant planets, not lower-mass planets like HD $77338 b$. However, population synthesis modeling is a statistical approach and gives general outcomes for the observed population. It does not preclude the formation of very low mass planets in metal-rich systems, but rather concludes that 

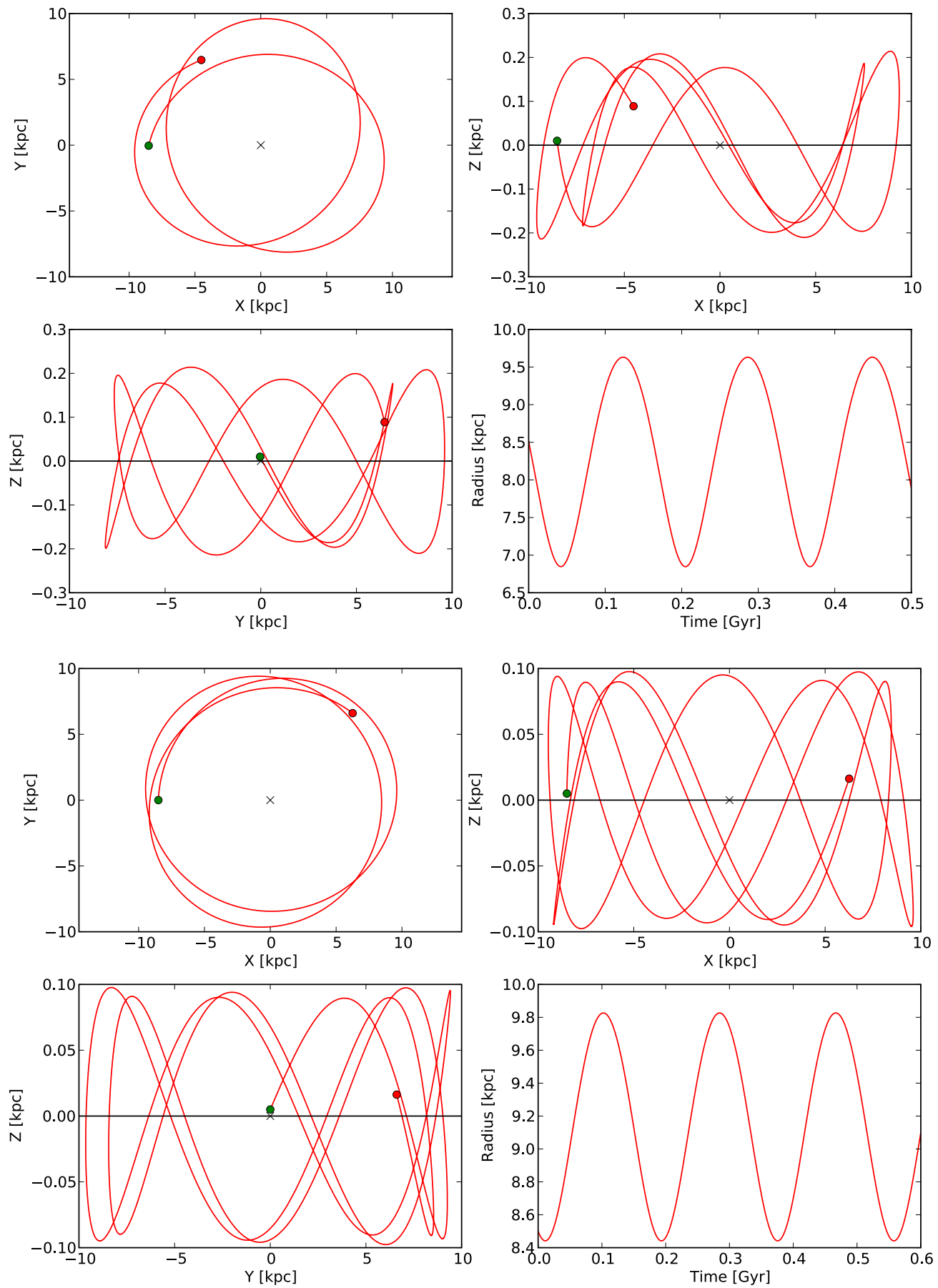

Figure 13. Calculated orbital motion of HD 77338 through the galaxy in all three spatial dimensions $X, Y, Z$, along with the distance from the galactic center are show as a function of time are shown in the top four panels, from top left to lower right, respectively. The same orbital calculations are shown in the lower four panels for the orbit of the Sun around the galaxy.

(A color version of this figure is available in the online journal.)

higher-mass planets are more likely around these stars in comparison to stars with a lower iron abundance.

The other elements are all found to have values of $[\mathrm{X} / \mathrm{Fe}]$ commensurate with that of the iron in HD 77338. Indeed, iron has long been used as a strong proxy for the overall metallicity $([\mathrm{M} / \mathrm{H}])$ of stars like the Sun. Therefore, for this system we might expect that any core in HD $77338 b$ is element heavy and possibly silicate rich. Sato et al. (2005) and Fortney et al. (2006) show that the transiting planet HD $149026 b$ must be around twothirds heavy elements by mass, and given that the star is super metal-rich, this points to a connection between the abundances of elements in stellar atmospheres and those of their retinue of planets. A further systematic search for the transit of HD $77338 b$ could help to shed light on this issue.

\section{MASS AND METALLICITY DISCUSSION}

HD 77338 is one of the most metal-rich stars currently known to host a planet. If we take the recent Trevisan et al. (2011) $[\mathrm{Fe} / \mathrm{H}]$ value of $+0.47 \pm 0.05$ dex for HD 77338 then only one 


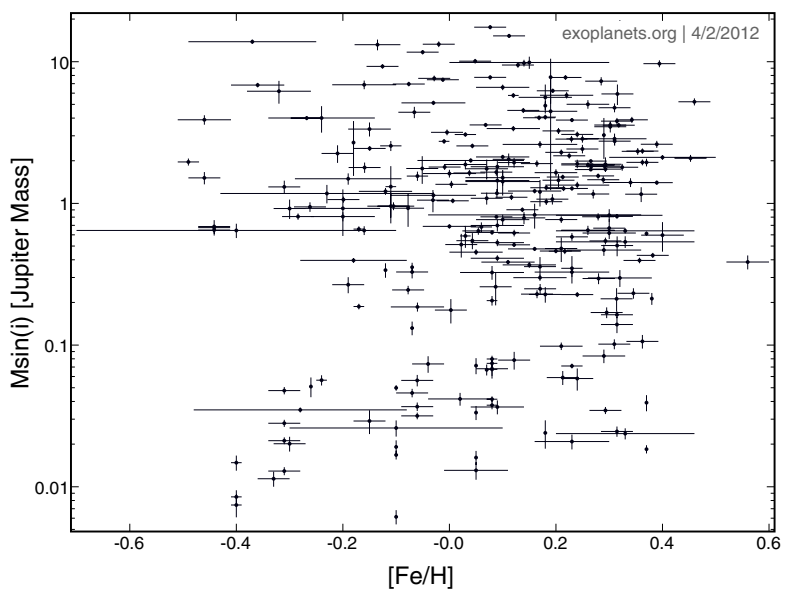

Figure 14. Plot of radial velocity detected planets host star metallicity against minimum mass of the planet, taken from the Exoplanet Database (Wright et al. 2011). Only planets around dwarf stars were included.

planet-host star has a metallicity higher than this, HD $126614 \mathrm{~A}$ with a metallicity of $+0.56 \pm 0.04$ dex (Howard et al. 2010). However, HD 126614 A has an M dwarf companion at only 33 AU separation from the host star, meaning HD $77338 b$ would be the most metal-rich single star known to host an exoplanet. Also, the low-mass nature of HD $77338 b\left(m \sin i<0.05 M_{\mathrm{J}}\right)$ helps to populate the low-mass metal-rich bin and shows that metal-rich, very low mass planets may be plentiful.

The high abundance of all the elements we have analyzed also indicates that the proto-planetary disk left over from the formation of HD 77338 was rich in planet-building material. The established preponderance for gas giant planets to favor metalrich stars (Gonzalez 1997; Fischer \& Valenti 2005; Sousa et al. 2011) can be seen in Figure 14. We also see that metal-rich stars tend to cover the entire phase space of planetary masses. Above a host star metallicity of $\sim 0.2$ dex there are planets covering the whole regime from low masses to high masses, in dense clusters. However, for lower metallicity systems, particularly at sub-solar metallicities, there are regions free from any planet detections, or at least less densely packed. This shows why metal-rich stars are so highly prized in the hunt for exoplanets and for better understanding the nature of planet formation and migration.

\subsection{Low-mass Systems}

Lower mass systems may show a somewhat different metallicity distribution in comparison to gas giants (Udry et al. 2006; Neves et al. 2009), with no clear metallicity bias. Models indicate that gas giant planets form outside the ice line boundary, taken to be $\sim 5 \mathrm{AU}$, in metal-poor disks (Mordasini et al. 2012) meaning smaller rocky/icy planets can form interior to this boundary, giving rise to the observed population of short period rocky planets around stars with a lower metal content.

Figure 15 shows the distribution of sub-Neptune mass planets ( $m \sin i \leqslant 0.05 M_{\mathrm{J}}$ ) as a function of metallicity using data taken from the Exoplanet Database ${ }^{9}$ as of 2012 February. All values we have used are listed in Table 5. One of the most striking features we see is the lack of planets with high metallicities and very low masses, in the lower right corner of the plot. Even though the numbers are relatively small at present, there appears a lower boundary that increases with metallicity, which would be contrary to the presumed biases in current radial velocity surveys

\footnotetext{
9 http://www.exoplanets.org
}

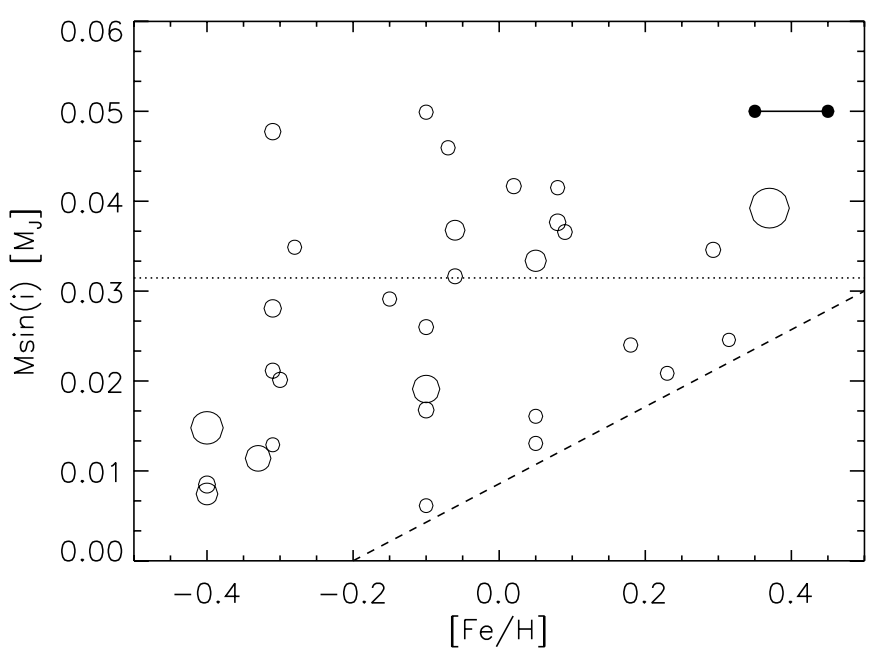

Figure 15. Metallicity against minimum mass for all radial velocity detected planets with a minimum mass less than that of Neptune $\left(m \sin i \leqslant 0.05 M_{\mathrm{J}}\right)$. The horizontal dotted line highlights the canonical boundary where runaway gas accretion onto the growing core is expected to occur. The dashed line marks the lower boundary in mass and the data points have been scaled in size by their orbital period. The filled circles connected by a straight line show the position of HD $77338 b$ using our metallicity and the metallicity of Trevisan et al. (2011), respectively.

given the number of metal-rich programs currently running. HD 77338 also appears to follow this trend given that it has a very high metallicity and a minimum mass commensurate with that of Uranus in our solar system.

A strong bias that could be manifest here is from the distribution of the orbital periods of the sample. Given the high fraction of short period gas giants in metal-rich systems, it is possible that lower-mass planets reside at longer orbital periods, meaning they will be biased against due to the well-known radial velocity bias against longer period and low amplitude objects (see Cumming 2004). To test this we scale the data points in Figure 15 by the ratio between each planet's period and that of the longest period planet in the set, GJ 876e. This shows us that this is not the case and that as expected, the planets are mostly short period planets at the perimeter of this boundary. It is also noticeable that beyond the cluster of planets near the perimeter of the boundary we mark by the dashed line, there is a possible valley in the metallicity plane, then the rest of the sample is found. A metallicity-mass correlation like this, at low-masses, would fit well with the core accretion model for planet formation, where the low-mass planets around super metal-rich stars attain a higher mass due to the abundance of planet forming material in the disk (Ida \& Lin 2004b; Mordasini et al. 2012). What is not obvious is why there would be a lower boundary. Possibly the planetesimals quickly accrete material and grow much faster than at lower metallicities, meaning the boundary traces the planet formation timescales for a given disk metallicity. In any case, if there is a lower boundary that holds to long period orbits for super metal-rich stars, this has consequences for the fraction of habitable Earth-mass planets in the galaxy, as super metal-rich stars will not be abundant in such planets.

\subsubsection{Monte Carlo Test}

To test if the lack of planets in the lower right corner of the metallicity-mass plane is statistically significant, and hence a metallicity-mass correlation for low-mass rocky planets is statistically significant, we perform a Monte Carlo analysis. 
Table 5

Orbital and Stellar Parameters for all Radial Velocity Detected Host Stars with sub-Neptune Mass Planets

\begin{tabular}{|c|c|c|c|c|c|c|c|c|c|c|}
\hline Planet & $\begin{array}{c}m \sin i \\
\left(M_{\mathrm{J}}\right)\end{array}$ & $\begin{array}{c}\text { Semimajor Axis } \\
(\mathrm{AU})\end{array}$ & $\begin{array}{l}\text { Period } \\
\text { (days) }\end{array}$ & Eccentricity & $\begin{array}{c}K \\
\left(\mathrm{~ms}^{-1}\right)\end{array}$ & $\begin{array}{c}M_{\star} \\
\left(M_{\odot}\right)\end{array}$ & $\begin{array}{l}{[\mathrm{Fe} / \mathrm{H}]} \\
(\mathrm{dex})\end{array}$ & $\begin{array}{c}V \\
(\mathrm{mag})\end{array}$ & SpT & $\begin{array}{c}U, V, W \\
\left(\mathrm{~km} \mathrm{~s}^{-1}\right)\end{array}$ \\
\hline GJ $3634 b^{1}$ & 0.022 & 0.029 & 2.65 & 0.08 & 5.57 & 0.45 & $\ldots$ & 11.90 & M2.5 & \\
\hline GJ $667 C b^{2}$ & 0.018 & 0.049 & 7.20 & 0.17 & 3.90 & 0.31 & -0.59 & 10.22 & M1.5V & $20,29,-27^{\mathrm{a}}$ \\
\hline GJ $667 \mathrm{C} c^{2}$ & 0.014 & 0.123 & 28.16 & $<0.27$ & 2.02 & 0.31 & -0.59 & 10.22 & M1.5V & $20,29,-27^{\mathrm{a}}$ \\
\hline HD $20794 b^{3}$ & 0.008 & 0.121 & 18.32 & 0.00 & 0.83 & 0.70 & -0.40 & 4.26 & G8V & $-79,-93,-29^{b}$ \\
\hline HD $20794 c^{3}$ & 0.007 & 0.204 & 40.11 & 0.00 & 0.56 & 0.70 & -0.40 & 4.26 & G8V & $-79,-93,-29^{b}$ \\
\hline HD $85512 b^{3}$ & 0.011 & 0.260 & 58.43 & 0.11 & 0.77 & 0.69 & -0.33 & 7.67 & $\mathrm{~K} 5 \mathrm{~V}$ & $34,11,-5^{\mathrm{b}}$ \\
\hline HD $4308 b^{4}$ & 0.048 & 0.119 & 15.56 & 0.00 & 4.07 & 0.93 & -0.31 & 6.55 & G3V & $50,-110,-27^{b}$ \\
\hline HD $40307 b^{5}$ & 0.013 & 0.047 & 4.31 & 0.00 & 1.97 & 0.74 & -0.31 & 7.17 & $\mathrm{~K} 2.5 \mathrm{~V}$ & $3,-25,-18^{\mathrm{b}}$ \\
\hline HD $40307 c^{5}$ & 0.021 & 0.080 & 9.62 & 0.00 & 2.47 & 0.74 & -0.31 & 7.17 & $\mathrm{~K} 2.5 \mathrm{~V}$ & $3,-25,-18^{\mathrm{b}}$ \\
\hline HD $40307 d^{5}$ & 0.028 & 0.132 & 20.46 & 0.00 & 2.55 & 0.74 & -0.31 & 7.17 & $\mathrm{~K} 2.5 \mathrm{~V}$ & $3,-25,-18^{b}$ \\
\hline HD $97658 b^{6}$ & 0.020 & 0.080 & 9.50 & 0.13 & 2.36 & 0.75 & -0.30 & 7.78 & K1V & $-11,-1,-2^{\mathrm{c}}$ \\
\hline GJ $674 b^{7}$ & 0.035 & 0.039 & 4.69 & 0.20 & 8.70 & 0.35 & -0.28 & 9.36 & $\mathrm{M} 2.5 \mathrm{~V}$ & $-15,-5,-19^{\mathrm{d}}$ \\
\hline GJ $176 b^{9}$ & 0.026 & 0.066 & 8.78 & 0.00 & 4.12 & 0.49 & -0.10 & 9.97 & $\mathrm{M} 2 \mathrm{~V}$ & $-26,-62,-13^{\mathrm{e}}$ \\
\hline GJ $581 b^{10}$ & 0.050 & 0.041 & 5.37 & 0.03 & 12.65 & 0.31 & -0.10 & 10.60 & M5 & $-25,-26,12^{\mathrm{d}}$ \\
\hline GJ $581 c^{10}$ & 0.017 & 0.073 & 12.92 & 0.07 & 3.18 & 0.31 & -0.10 & 10.60 & M5 & $-25,-26,12^{\mathrm{d}}$ \\
\hline GJ $581 d^{10}$ & 0.019 & 0.218 & 66.64 & 0.25 & 2.16 & 0.31 & -0.10 & 10.60 & M5 & $-25,-26,12^{\mathrm{d}}$ \\
\hline GJ $581 e^{10}$ & 0.006 & 0.028 & 3.15 & 0.32 & 1.96 & 0.31 & -0.10 & 10.60 & M5 & $-25,-26,12^{\mathrm{d}}$ \\
\hline BD-082823 $b^{11}$ & 0.046 & 0.056 & 5.60 & 0.15 & 6.50 & 0.74 & -0.07 & 9.99 & $\mathrm{~K} 3 \mathrm{~V}$ & $\ldots$ \\
\hline HD $69830 b^{4}$ & 0.032 & 0.078 & 8.67 & 0.10 & 3.51 & 0.85 & -0.06 & 5.95 & $\mathrm{~K} 0 \mathrm{~V}$ & $29,-61,-10^{\mathrm{b}}$ \\
\hline HD $69830 c^{4}$ & 0.037 & 0.185 & 31.56 & 0.13 & 2.66 & 0.85 & -0.06 & 5.95 & K0V & $29,-61,-10^{\mathrm{b}}$ \\
\hline HD $125595 b^{12}$ & 0.042 & 0.081 & 9.67 & 0.00 & 4.79 & 0.76 & 0.02 & 9.03 & $\mathrm{~K} 3 / \mathrm{K} 4 \mathrm{~V}$ & $-34,-42,9^{\mathrm{e}}$ \\
\hline $61 \mathrm{Vir} b^{13}$ & 0.016 & 0.050 & 4.21 & 0.12 & 2.12 & 0.94 & 0.05 & 4.87 & G5V & $-24,-47,-32^{\mathrm{b}}$ \\
\hline $61 \operatorname{Vir} c^{13}$ & 0.033 & 0.217 & 38.02 & 0.14 & 2.12 & 0.94 & 0.05 & 4.87 & G5V & $-24,-47,-32^{\mathrm{b}}$ \\
\hline HD $156668 b^{14}$ & 0.013 & 0.050 & 4.65 & 0.00 & 1.89 & 0.77 & 0.05 & 8.42 & $\mathrm{~K} 3 \mathrm{~V}$ & $\ldots$ \\
\hline HD $10180 c^{15}$ & 0.042 & 0.064 & 5.76 & 0.08 & 4.54 & 1.06 & 0.08 & 7.33 & G1V & $9,-16,-30^{\mathrm{b}}$ \\
\hline HD $10180 d^{15}$ & 0.038 & 0.129 & 16.36 & 0.14 & 2.93 & 1.06 & 0.08 & 7.33 & G1V & $9,-16,-30^{\mathrm{b}}$ \\
\hline HIP57274b $b^{16}$ & 0.037 & 0.071 & 8.14 & 0.19 & 4.64 & 0.73 & 0.09 & 8.96 & K8 & $2,-34,27^{\mathrm{e}}$ \\
\hline$\mu \operatorname{Ara} d^{4}$ & 0.035 & 0.093 & 9.64 & 0.17 & 3.06 & 1.15 & 0.29 & 5.12 & G3V & $-15,-7,-3^{\mathrm{e}}$ \\
\hline $55 \mathrm{Cnc} e^{10}$ & 0.025 & 0.015 & 0.74 & 0.06 & 5.92 & 0.90 & 0.31 & 5.96 & G8V & $-37,-18,-8^{\mathrm{d}}$ \\
\hline GJ $876 d^{19}$ & 0.018 & 0.021 & 1.94 & 0.21 & 6.56 & 0.32 & 0.37 & 10.20 & M5 & $-13,-20,-12^{\mathrm{d}}$ \\
\hline GJ $876 e^{19}$ & 0.039 & 0.333 & 124.26 & 0.05 & 3.42 & 0.32 & 0.37 & 10.20 & M5 & $-13,-20,-12^{\mathrm{d}}$ \\
\hline
\end{tabular}

Notes. ${ }^{1}$ Bonfils et al. (2011); ${ }^{2}$ Anglada-Escudé et al. (2012); ${ }^{3}$ Pepe et al. (2011); ${ }^{4}$ Valenti \& Fischer (2005); ${ }^{5}$ Mayor et al. (2009); ${ }^{6}$ Henry et al. (2011); ${ }^{7}$ Bonfils et al. (2007); ${ }^{8}$ Howard et al. (2009); ${ }^{9}$ Forveille et al. (2009); ${ }^{10}$ von Braun et al. (2011); ${ }^{11}$ Hébrard et al. (2010); ${ }^{12}$ Ségransan et al. (2011); ${ }^{13}$ Vogt et al. (2010); ${ }^{14}$ Howard et al. (2011); ${ }^{15}$ Lovis et al. (2011); ${ }^{16}$ Fischer et al. (2012); ${ }^{17}$ Rivera et al. (2010); ${ }^{18}$ Lo Curto et al. (2010); ${ }^{19}$ Johnson \&

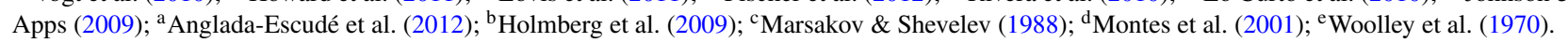

We setup the model in two parts where we first generate a random realization of the sample of 36 low-mass planets we have tested in Figure 15. To generate the masses in a robust fashion we take the observationally driven mass distribution for lower-mass planets (Butler et al. 2006; Cumming et al. 2008). Lopez \& Jenkins (2012) show that a power-law model such as this explains the observed turnover of the mass distribution at the lowest masses and hence the model is a robust representation of the current distribution of exoplanets. Equation (3) shows the form of the mass function we consider:

$$
d N / d M=k M^{-1.2}
$$

Here $d N / d M$ represents the frequency of planets as a function of mass, $M$ is the mass bin considered, and $k$ is a scaling constant to fit the observed population. We can then integrate this mass function equation (Equation (4)) to get the result shown in Equation (5), and by taking the inverse we can generate the cumulative distribution function (CDF; Equation (6)) that can be used to draw masses randomly from the observed population of exoplanets:

$$
\begin{gathered}
d N=k \int_{-\infty}^{M_{o}} M^{-1.2} d M \\
N=\frac{-5 k}{M^{0.2}} .
\end{gathered}
$$

Now if we set the constant $k$ equal to -0.014 this will normalize the function such that we have a PDF and the corresponding $\mathrm{CDF}$ is given by

$$
\operatorname{CDF}(M)=\left(\frac{0.069}{N}\right)^{5}
$$

The CDF allows us to draw random masses for planets and restrict the values to be within the range between 0.006 and $0.050 M_{\mathrm{J}}$ to match our region of interest. We can then randomly generate metallicities, and in this test we simply draw from a uniform distribution of $[\mathrm{Fe} / \mathrm{H}]$ values in the range from -0.5 to +0.5 dex, which covers all the values of metallicities in our test. 
Once we have built a random sample of planets we can then add the boundary and test how many times there are planets that reside under the boundary region we identify in Figure 15. We run the code 1,000,000 times to ensure a high level of robustness in the final probability measurement. Our test reveals that for a sample of 36 stars, assuming a uniform metallicity distribution, $99.9993 \%$ of the time there are planets to be found below the boundary region. Therefore, this low-mass planet desert is statistically significant at almost the $\sim 4.5 \sigma$ level, under these test conditions. For a sample of only 10 planets we still find that $96 \%$ of the time there are planets below the boundary region, and for a sample of five planets we find a percentage of $81 \%$. These tests indicate that there is some significant correlation between metallicity and mass for low-mass planets, at least in the metal-rich regime, that would be important to quantify in the future.

We do note that our results can vary due to the assumed metallicity model that we use to draw the sample of random metallicities from. For instance, the result will become more significant for a distribution that follows the current observed distribution for more massive exoplanets (see Sousa et al. 2011), and vice versa for a distribution shaped in the opposite fashion.

As for the possible valley, the sample is as yet too small to draw statistically significant conclusions and therefore difficult to test without detailed modeling of the core accretion method of forming planets, and therefore we are only pointing out the possibility that there are two classes of low-mass planets that have a metallicity dependency. In particular, magnitude or distance limited samples will be biased toward sub-solar metallicities as they will be governed by the metallicity distribution of the local galactic neighborhood (Holmberg et al. 2009). However, to fully probe these samples it is necessary to simulate the radial velocity data for each star individually (O'Toole et al. 2009) to better understand the completeness of the bins we have discussed.

\subsubsection{What does Kepler Say?}

The Kepler Space Telescope has recently released a plethora of transiting planet candidates, that range in radius from below Earth radii up into the gas giant planet arena (Borucki et al. 2011). Follow-up of Kepler targets are difficult due to the relative faintness of the candidate stars; however, Buchhave et al. (2012) have published the first large set of spectroscopic metallicity for 156 Kepler planet-host stars that can be used to draw statistically significant conclusions on Kepler host star abundance patterns.

In Figure 16 we show the Kepler results published in Buchhave et al. in the metallicity-radius plane. We only show the stars that host planets with radii of $4 R_{\oplus}$ or less, and we do not distinguish between multi-planet systems and single planet-host stars. The $4 R_{\oplus}$ limit was chosen simply because typical massto-radius relationships, e.g., $M_{\mathrm{p}}=R_{\mathrm{p}}^{2.06}$ (Lissauer et al. 2011), would give rise to around the Neptune-mass limit highlighted in Figure 15 . The paucity of planets we find in the metallicity-mass plane from the radial velocity data could therefore be manifest in the Kepler data too. However, we note that a single massto-radius relationship is surely unrealistic due to the possible diversity of rocky planets (see Seager et al. 2007).

Studying the lower right corner of Figure 16, the high metallicity and low-mass regime, we do find a paucity of planets when we compared to the lower left corner, or the low metallicity and low-mass region. To better highlight this relative planet desert we bound the region by a dashed box and also a similar rising boundary line (solid curve) as we show in the metallicity-mass plane. The Kepler data are in good agreement

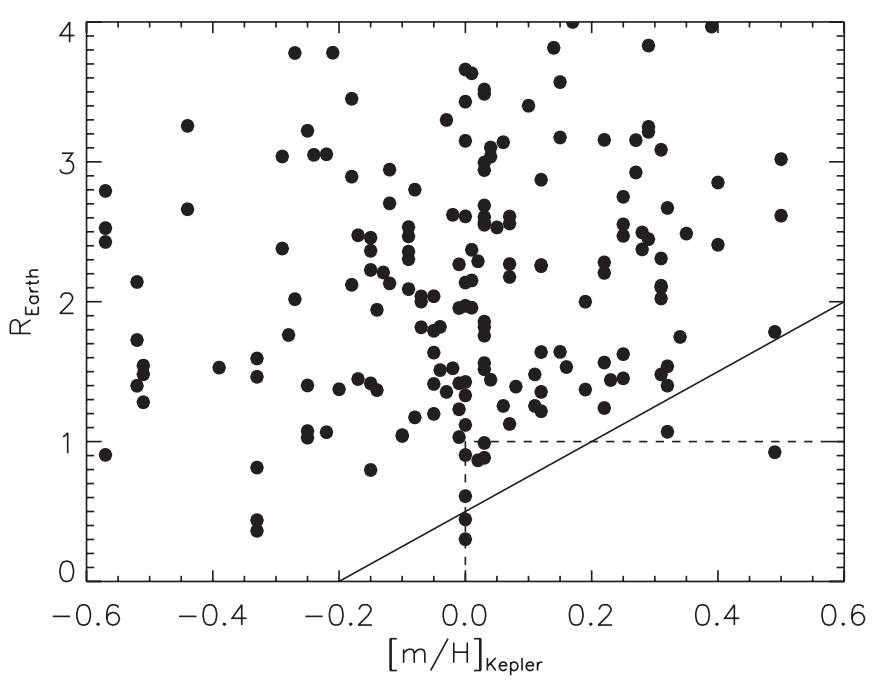

Figure 16. Kepler results for 156 planet-hosting stars detected by Kepler in the metallicity-radius plane. The dashed box region in the lower right corner highlights the lack of low-mass Kepler transits in comparison to the more metalpoor region.

with the radial velocity sample with respect to this paucity of planets, although not likely in absolute value, with only two planets significantly below the boundary region we highlight. In fact, within the boxed region, parameterized by the host star having a metallicity of solar or above and the transiting planet having an Earth radius or lower, there are only eight planets, and only one of these planets is currently in a single system. Therefore, it could be that really low-mass planets can exist in the super metal-rich regime if they are part of multiplanet systems, since under core accretion the planetesimals will compete for material to form and this will mean less material for each of the planetesimals to reach higher core masses.

If these features however are real, then for a given stellar/ disk metallicity it appears that planetesimals quickly grow to their boundary region and stop there, or they then quickly make the transition to around 2-3 times their boundary mass and either continue growing to higher-mass planets before the disk dissipates, or stay where they are due to depletion of the disk material.

We thank the anonymous referee for useful suggestions and prompt response. We thank Rene Mendez for useful discussions on the kinematic orbits. We also thank Claudio Melo for help in re-reducing the old HARPS data with the new DRS. J.S.J. also acknowledges funding by Fondecyt through grant 3110004 and partial support from Centro de Astrofísica FONDAP 15010003, the GEMINI-CONICYT FUND and from the Comité Mixto ESO-GOBIERNO DE CHILE. H.R.A.J., M.T., F.M., Y.V.P., O.I., and D.J.P. are supported by RoPACS, a Marie Curie Initial Training Network funded by the European Commission's Seventh Framework Programme. P.R. acknowledges support through Fondecyt grant 1120299. A.J. acknowledges support from Fondecyt project 1095213 and from the Millennium Science initiative, Chilean Ministry of Economy (Nuclei P10-022-F and P07-021-F). A.D.J. is funded by a Fondecyt postdoctorado, under project number 3100098. M.T.R. received support from CATA (PB06 Conicyt). Based on observations made with the European Southern Observatory telescopes obtained from the ESO/ST-ECF Science Archive Facility. We also acknowledge use of the Simbad and Vizier databases. 


\section{REFERENCES}

Abadi, M. G., Navarro, J. F., Steinmetz, M., \& Eke, V. R. 2003, ApJ, 591, 499 Anglada-Escudé, G., Arriagada, P., Vogt, S. S., et al. 2012, ApJL, 751, L16 Barbuy, B., \& Grenon, M. 1990, in European Southern Observatory Conference and Workshop Proceedings, Vol. 35, ESO/CTIO Workshop on Bulges of Galaxies, ed. B. J. Jarvis \& D. M. Terndrup, 83

Bean, J. L., Désert, J.-M., Kabath, P., et al. 2011, ApJ, 743, 92

Beaulieu, J. P., Kipping, D. M., Batista, V., et al. 2010, MNRAS, 409, 963

Bensby, T., Feltzing, S., Lundström, I., \& Ilyin, I. 2005, A\&A, 433, 185

Boisse, I., Bouchy, F., Hébrard, G., et al. 2011, A\&A, 528, A4

Bonfils, X., Gillon, M., Forveille, T., et al. 2011, A\&A, 528, A111

Bonfils, X., Mayor, M., Delfosse, X., et al. 2007, A\&A, 474, 293

Borucki, W. J., Koch, D. G., Basri, G., et al. 2011, ApJ, 736, 19

Bovy, J., Rix, H.-W., \& Hogg, D. W. 2012a, ApJ, 751, 131

Bovy, J., Rix, H.-W., Hogg, D. W., et al. 2012b, ApJ, 755, 115

Brook, C. B., Kawata, D., Gibson, B. K., \& Freeman, K. C. 2004, ApJ, 612, 894

Buchhave, L. A., Latham, D. W., Johansen, A., et al. 2012, Natur, 486, 375

Butler, R. P., Wright, J. T., Marcy, G. W., et al. 2006, ApJ, 646, 505

Cenarro, A. J., Peletier, R. F., Sánchez-Blázquez, P., et al. 2007, MNRAS, 374,664

Cumming, A. 2004, MNRAS, 354, 1165

Cumming, A., Butler, R. P., Marcy, G. W., et al. 2008, PASP, 120, 531

Demarque, P., Woo, J.-H., Kim, Y.-C., \& Yi, S. K. 2004, ApJS, 155, 667

Eggen, O. J. 1969, PASP, 81, 553

Feroz, F., Balan, S. T., \& Hobson, M. P. 2011, MNRAS, 415, 3462

Fischer, D. A., Gaidos, E., Howard, A. W., et al. 2012, ApJ, 745, 21

Fischer, D. A., \& Valenti, J. 2005, ApJ, 622, 1102

Fortney, J. J., Saumon, D., Marley, M. S., Lodders, K., \& Freedman, R. S. 2006, ApJ, 642, 495

Forveille, T., Bonfils, X., Delfosse, X., et al. 2009, A\&A, 493, 645

Gonzalez, G. 1997, MNRAS, 285, 403

Grevesse, N., \& Sauval, A. J. 1998, SSRv, 85, 161

Haario, H., Saksman, E., \& Tamminen, J. 2001, Bernoulli, 7, 223

Hébrard, G., Udry, S., Lo Curto, G., et al. 2010, A\&A, 512, A46

Henry, G. W., Howard, A. W., Marcy, G. W., Fischer, D. A., \& Johnson, J. A. 2011, arXiv:1109.2549

Holmberg, J., Nordström, B., \& Andersen, J. 2009, A\&A, 501, 941

Howard, A. W., Johnson, J. A., Marcy, G. W., et al. 2009, ApJ, 696, 75

Howard, A. W., Johnson, J. A., Marcy, G. W., et al. 2010, ApJ, 721, 1467

Howard, A. W., Johnson, J. A., Marcy, G. W., et al. 2011, ApJ, 730, 10

Ida, S., \& Lin, D. N. C. 2004a, ApJ, 604, 388

Ida, S., \& Lin, D. N. C. 2004b, ApJ, 616, 567

Isaacson, H., \& Fischer, D. 2010, ApJ, 725, 875

Jehin, E., Gillon, M., Chantry, V., \& Magain, P. 2010, Ciel, 72, 326

Jenkins, J. S., Jones, H. R. A., Goździewski, K., et al. 2009, MNRAS, 398, 911

Jenkins, J. S., Jones, H. R. A., Pavlenko, Y., et al. 2008, A\&A, 485, 571

Jenkins, J. S., Jones, H. R. A., Tinney, C. G., et al. 2006, MNRAS, 372, 163

Jenkins, J. S., \& Jordan, A. 2011, in ASP Conf. Ser. 448, 16th Cambridge

Workshop on Cool Stars, Stellar Systems, and the Sun, ed. C. Johns-Krull (San Francisco, CA: ASP), 991

Jenkins, J. S., Murgas, F., Rojo, P., et al. 2011, A\&A, 531, A8

Jenkins, J. S., Pavlenko, Y. V., Ivanyuk, O., et al. 2012, MNRAS, 420, 3587
Johnson, J. A., \& Apps, K. 2009, ApJ, 699, 933

Kass, R. E., \& Raftery, A. E. 1995, JASA, 430, 773

Lin, D. N. C., \& Papaloizou, J. 1986, ApJ, 309, 846

Lissauer, J. J., Ragozzine, D., Fabrycky, D. C., et al. 2011, ApJS, 197, 8

Lo Curto, G., Mayor, M., Benz, W., et al. 2010, A\&A, 512, A48

Lopez, S., \& Jenkins, J. S. 2012, ApJ, 756, 177

Lovis, C., Ségransan, D., Mayor, M., et al. 2011, A\&A, 528, A112

Lucy, L. B., \& Sweeney, M. A. 1971, AJ, 76, 544

Marsakov, V. A., \& Shevelev, Y. G. 1988, BICDS, 35, 129

Mayor, M., Bonfils, X., Forveille, T., et al. 2009, A\&A, 507, 487

Mayor, M., Pepe, F., Queloz, D., et al. 2003, Msngr, 114, 20

Montes, D., López-Santiago, J., Gálvez, M. C., et al. 2001, MNRAS, 328, 45

Mordasini, C., Alibert, Y., \& Benz, W. 2009, A\&A, 501, 1139

Mordasini, C., Alibert, Y., Benz, W., Klahr, H., \& Henning, T. 2012, A\&A, 541,97

Neves, V., Santos, N. C., Sousa, S. G., Correia, A. C. M., \& Israelian, G. 2009, A\&A, 497, 563

Nissen, P. E., \& Schuster, W. J. 2010, A\&A, 511, L10

O'Toole, S. J., Tinney, C. G., \& Jones, H. R. A. 2008, MNRAS, 386, 516

O’Toole, S. J., Tinney, C. G., Jones, H. R. A., et al. 2009, MNRAS, 392, 641

Pavlenko, Y. V., Jenkins, J. S., Jones, H. R. A., Ivanyuk, O., \& Pinfield, D. J. 2012, MNRAS, 422, 542

Pepe, F., Lovis, C., Ségransan, D., et al. 2011, A\&A, 534, A58

Pepe, F., Mayor, M., Queloz, D., et al. 2004, A\&A, 423, 385

Perryman, M. A. C., Lindegren, L., Kovalevsky, J., et al. 1997, A\&A, 323, L49

Pont, F., Knutson, H., Gilliland, R. L., Moutou, C., \& Charbonneau, D. 2008, MNRAS, 385, 109

Queloz, D., Henry, G. W., Sivan, J. P., et al. 2001, A\&A, 379, 279

Rivera, E. J., Butler, R. P., Vogt, S. S., et al. 2010, ApJ, 708, 1492

Santos, N. C., Gomes da Silva, J., Lovis, C., \& Melo, C. 2010, A\&A, 511, A54

Sato, B., Fischer, D. A., Henry, G. W., et al. 2005, ApJ, 633, 465

Scholz, R.-D., Odenkirchen, M., Hirte, S., et al. 1996, MNRAS, 278, 251

Seager, S., Kuchner, M., Hier-Majumder, C. A., \& Militzer, B. 2007, ApJ, 669,1279

Ségransan, D., Mayor, M., Udry, S., et al. 2011, A\&A, 535, A54

Sousa, S. G., Santos, N. C., Israelian, G., Mayor, M., \& Udry, S. 2011, A\&A, 533, A141

Statler, T. S. 1988, ApJ, 331, 71

Swain, M. R., Tinetti, G., Vasisht, G., et al. 2009, ApJ, 704, 1616

Taylor, B. J. 2005, ApJS, 161, 444

Trevisan, M., Barbuy, B., Eriksson, K., et al. 2011, A\&A, 535, A42

Tuomi, M. 2011, A\&A, 528, L5

Tuomi, M., Jones, H. R., Jenkins, J. S., et al. 2013, A\&A, 551, 79

Tuomi, M., Pinfield, D., \& Jones, H. R. A. 2011, A\&A, 532, A116

Udry, S., Mayor, M., Benz, W., et al. 2006, A\&A, 447, 361

Valenti, J. A., \& Fischer, D. A. 2005, ApJS, 159, 141

van Leeuwen, F. 2007, A\&A, 474, 653

Villalobos, Á., \& Helmi, A. 2008, MNRAS, 391, 1806

Vogt, S. S., Butler, R. P., Rivera, E. J., et al. 2010, ApJ, 723, 954

von Braun, K., Boyajian, T. S., ten Brummelaar, T. A., et al. 2011, ApJ, 740,49

Woolley, R., Epps, E. A., Penston, M. J., \& Pocock, S. B. 1970, ROAn, 5, 227

Wright, J. T., Fakhouri, O., Marcy, G. W., et al. 2011, PASP, 123, 412 